\author{
Marquette University \\ e-Publications@Marquette
}

$4-2017$

\title{
Nutrition Labeling in the United States and the Role of Consumer Processing, Message Structure, and Moderating Conditions
}

J. Craig Andrews

Marquette University, craig.andrews@marquette.edu

Scot Burton

University of Arkansas - Main Campus

Laurel Aynne Cook

West Virginia University

Follow this and additional works at: https://epublications.marquette.edu/market_fac

Part of the Marketing Commons

\section{Recommended Citation}

Andrews, J. Craig; Burton, Scot; and Cook, Laurel Aynne, "Nutrition Labeling in the United States and the Role of Consumer Processing, Message Structure, and Moderating Conditions" (2017). Marketing Faculty Research and Publications. 263.

https://epublications.marquette.edu/market_fac/263 


\section{Oxford Research Encyclopedia of Communication}

\section{Nutrition Labeling in the United States and the Role of Consumer Processing, Message Structure, and Moderating Conditions}

J. Craig Andrews, Scot Burton, and Laurel Aynne Cook

Subject: Health and Risk Communication Online Publication Date: Apr 2017

DOI: $10.1093 /$ acrefore/9780190228613.013.546

\section{Summary and Keywords}

It has been since 1990 that the landmark Nutritional Labeling Education Act (NLEA) was passed in the United States, and since 1969 that the first White House Conference on Food, Nutrition and Health occurred. In the time since these important events, considerable research has been conducted on how U.S. consumers process and use nutritional labeling. An up-to-date review of nutritional labeling research must address key findings on the processing and use of nutrition facts panels (NFPs), restaurant labeling, front-of-pack (FOP) symbols, health and nutrient content claims, new labeling efforts (e.g., for meat products), and claims not regulated by the U.S. Food and Drug Administration (FDA). Message structure mediates the ways in which consumers process nutritional labeling while moderating conditions affect research outcomes associated with labeling efforts.

The most recent policy issues and problems to be considered (e.g., by the FDA) include nutritional labeling as well as identifying opportunities for consumer research in helping to promote healthy lifestyles and reducing obesity in the United States and throughout the world. For example, several unanswered research questions remain regarding how the proposed changes to the NFPs-beef, poultry, and seafood labeling; restaurant chain calorie labeling; alternative FOP formats; and regulated and unregulated health and nutrient content claims-will affect consumers. Researchers have yet to examine not only these different labeling and nutrition information formats, but also how they might interact with one another and the role of key moderating conditions (e.g., one's motivation, ability opportunity to process nutrition information) in affecting consumer processing and behavior. 


\section{Introduction}

It has been since 1969 that the first White House Conference on Food, Nutrition and Health in the United States took place encouraging greater nutrition education and healthy lifestyle changes to help prevent chronic diseases, such as hypertension, coronary heart disease, diabetes, and cancer. It also has been since 1990 that the Nutrition Labeling Health \& Education Act (NLEA, 1990) was passed in the United States, requiring the first standardized nutrition facts panel (NFP) in the world. Considerable research has been conducted on nutrition labeling in the U.S. since these events, and in this article we present key research findings on consumer processing, message structure, and moderating conditions affecting nutritional labeling efforts. We also consider recent mandates, forthcoming changes in nutrition labeling, and opportunities for future research.

Sections covered in this chapter include: (1) history of the NFPs, testing of alternative formats, role of moderating conditions (e.g., motivation, knowledge), and recent updates; (2) restaurant labeling, research, and moderating conditions; (3) front-of-pack (FOP) symbols, research on reductive/nutrient-specific versus evaluative/summary formats, and moderating conditions; (4) health and nutrient content claim research, including consumer processing issues (e.g., halo effects) and moderating conditions; (5) new labeling efforts (e.g., meat products); and (6) some claims not currently regulated by the U.S. FDA (e.g., organic, natural, gluten-free, GMO-free). Important aspects of consumer processing (e.g., prior beliefs, cognition versus affect/emotion, halos, habits, receiver characteristics), message structure (e.g., imagery/cues, framing and priming, risk disclosures), and moderating conditions (e.g., motivation, knowledge, literacy, opportunity to process) will be applied in examining the nutrition labeling research examples presented. We conclude with a final section discussing future policy issues and problems associated with U.S. nutrition labeling efforts in encouraging healthy lifestyles and in reducing obesity. 


\section{Nutrition Facts Panels}

\section{Brief History of Nutrition Labeling and NFPs in the United States}

The first NFP in the world had its beginnings with a White House Conference on Food, Nutrition and Health (1970) that focused not only on reducing hunger and malnutrition in the United States, but also on improving nutrition education and the accuracy of nutrition ingredients listed voluntarily on food packages. Following this conference, the U.S. FDA adopted voluntary nutrition labeling requirements in 1973 which focused on deficiencies in essential vitamins and minerals. Nutritional labels were mandatory only on fortified foods or if claims were made about the nutritional properties of the foods (Derby \& Levy, 2001). Yet, several years later, a report on nutritional labeling (Porter \& Earl, 1990) identified the following major deficiencies with the voluntary food labeling program in the United States: (1) only 40\% of FDA-regulated packaged foods had labeling, (2) deficiencies in nutrient content information disclosed (e.g., saturated fat, cholesterol, and fiber versus vitamins and minerals that were by then not deficient), (3) misleading label claims of nutrient content, and (4) a lack of standardized definitions (Derby \& Levy, 2001). Also, in the 1980s, health claims (i.e., linking a nutrient with a disease) were not allowed on food packaging unless they satisfied the standard for drugs. The Kellogg Company then bypassed the FDA with its use of a National Cancer Institute health claim for fiber and cancer for its All-Bran cereal. As a result, and combined with court decisions, nutrient content and health claims became rampant in the late 1980s.

The NLEA of 1990 helped to address these deficiencies and provided the FDA with the authority to require all packaged foods to bear nutrition labeling (i.e., nutrition facts panels) and require that all nutrient content claims (e.g., "high fiber," "low sodium," etc.) and health claims (e.g., "a diet low in total fat may reduce the risk of cancers") be consistent with agency regulations based on public health evidence. The overall objective of the NLEA was to provide information that could aid consumers in making healthy lifestyle changes in helping to prevent chronic diseases, such as hypertension, coronary heart disease, diabetes, and cancer. The major provisions of the NLEA, published by the FDA on January 6, 1993 (FDA, 1994), include the following:

- Required and standardized labeling (NFPs) for nearly all packaged foods.

- Per-serving information on key nutrients of public health concern (e.g., at that time, fat, saturated fat, cholesterol, sodium, dietary fiber).

- Percent dally values (\%DVs) to aid consumers in placing the food's nutrient information in the context of a total daily diet (based on 2,000 calories).

- Uniform definitions for nutrient content claims, such as "low," "light," "reduced," and "lean." 


\section{Nutrition Labeling in the United States and the Role of Consumer Processing, Message Structure, and Moderating Conditions}

- A limited and strictly-defined set of allowable health claims describing the relationship between a food's nutrients (e.g., calcium) and a disease (e.g., osteoporosis).

\section{Consumer Research on NFPs}

The original objectives for the NFPs were to contribute to nutrition education and to assist consumers in dietary management (Derby \& Levy, 2001). Thus, some key outcomes for these objectives include NFP exposure, awareness, comprehension, product evaluations, attitude change, intentions, and/or behavior. The challenge in assessing behavior as an outcome is that dietary choices and purchase behavior can be a result of a multitude of factors beyond nutritional labeling, including pricing, product availability, brand equity and consumer loyalty, promotion and advertising, prior attitudes toward nutrition, and consumer motivation, and ability and opportunity to process nutrition information. Following the implementation of the NFPs in 1994, the FDA's Food Labeling and Packaging Survey (FLAPS), FDA's Food Label Use and Education Surveys (FLUENES), and the FDA Health and Diet Surveys examined the NFP's self-reported awareness and use among consumers (Derby \& Levy, 2001). For example, in the FLUENES, $72 \%$ of those who noticed the NFP rated it as somewhat better or much better than the previous, voluntary label. Also, for those who used the food label, the highest percentage of specific use (at 62\%) was to "see how high or low a food is in a specific nutrient." Finally, the FDA Diet and Health Survey showed that a majority of pre-NLEA consumers reported using the old label the first time they purchased a food product, and this rose to three-quarters of consumers for the post-NLEA NFPs.

Yet, in order to examine cause-and-effect relationships (Cook \& Campbell, 1979), we now summarize the findings of a selected group of 35 consumer studies on the NFPs, almost all using experimental designs (these appear in chronological order in Table 1). The review of nutritional labeling research in Hieke and Taylor (2012) serves as an initial basis for the selection of these important studies from the consumer research discipline. We also searched major marketing and public health journals that publish nutritional labeling research for studies to be included in Table 1. 
Table 1 Overview of Studies Examining Effects of Nutrition Facts Panels

\begin{tabular}{|c|c|c|c|c|c|c|}
\hline $\begin{array}{l}\text { Authors/Date } \\
\text { of Study }\end{array}$ & Study Design & Manipulations & Study Focus & Study Sample & $\begin{array}{l}\text { Dependent } \\
\text { Variable(s) }\end{array}$ & $\begin{array}{l}\text { Study } \\
\text { Findings }\end{array}$ \\
\hline $\begin{array}{l}\text { Asam and } \\
\text { Bucklin (1973) }\end{array}$ & $\begin{array}{l}\text { Latin Square } \\
\text { experiment }\end{array}$ & $\begin{array}{l}\text { Four assigned } \\
\text { nutrition levels }\end{array}$ & $\begin{array}{l}\text { Brands of } \\
\text { canned peas }\end{array}$ & $\begin{array}{l}200 \text { mall } \\
\text { patrons at four } \\
\text { locations }\end{array}$ & $\begin{array}{l}\text { Shopper } \\
\text { perceptions } \\
\text { and purchase } \\
\text { preferences }\end{array}$ & $\begin{array}{l}\text { Detailed labels } \\
\text { (e.g., each } \\
\text { serving } \\
\text { contains } 0.4 \\
\text { grams of fat) } \\
\text { led to } \\
\text { significantly } \\
\text { better shopper } \\
\text { perceptions } \\
\text { and purchase } \\
\text { preferences } \\
\text { than more } \\
\text { ambiguous } \\
\text { labels (e.g., } \\
\text { "low in fat") }\end{array}$ \\
\hline
\end{tabular}




\begin{tabular}{|c|c|c|c|c|c|c|}
\hline $\begin{array}{l}\text { Lenahan et al. } \\
\text { (1973) }\end{array}$ & $\begin{array}{l}\text { Survey, } \\
\text { shopper } \\
\text { interviews }\end{array}$ & $\begin{array}{l}\text { Presentation of } \\
\text { different } \\
\text { nutrition label } \\
\text { formats }\end{array}$ & $\begin{array}{l}\text { General survey } \\
\text { and shopper } \\
\text { interviews }\end{array}$ & $\begin{array}{l}\text { National } \\
\text { probability } \\
\text { sample of } 2,195 \\
\text { adults and } \\
4,435 \text { shopper } \\
\text { interviews }\end{array}$ & $\begin{array}{l}\text { Consumer } \\
\text { perceptions } \\
\text { and } \\
\text { information } \\
\text { usage }\end{array}$ & $\begin{array}{l}\text { Significant } \\
\text { majority } \\
\text { preferred \% } \\
\text { RDA format; } \\
\text { overall use is } \\
\text { small, yet large } \\
\text { portion of those } \\
\text { who saw/ } \\
\text { understood } \\
\text { labels used } \\
\text { them (59\%) }\end{array}$ \\
\hline $\begin{array}{l}\text { Jacoby et al. } \\
(1977)\end{array}$ & $\begin{array}{l}\text { Purchase } \\
\text { decision tasks } \\
\text { from product } \\
\text { arrays }\end{array}$ & $\begin{array}{l}\text { Presentation of } \\
\text { product arrays } \\
\text { containing } \\
\text { nutritional } \\
\text { information } \\
\text { values }\end{array}$ & $\begin{array}{l}\text { Cereal and } \\
\text { margarine } \\
\text { products }\end{array}$ & $\begin{array}{l}\text { Six studies, } \\
\text { ranging from } \\
60 \text { students up } \\
\text { to } 480 \\
\text { shoppers }\end{array}$ & $\begin{array}{l}\text { Nutrition value } \\
\text { acquisition and } \\
\text { comprehension; } \\
\text { objective } \\
\text { nutrition } \\
\text { knowledge } \\
\text { measured }\end{array}$ & $\begin{array}{l}\text { Most } \\
\text { consumers did } \\
\text { not use nor } \\
\text { comprehend } \\
\text { nutritional } \\
\text { information in } \\
\text { making food } \\
\text { purchase } \\
\text { decisions }\end{array}$ \\
\hline
\end{tabular}




\begin{tabular}{|c|c|c|c|c|c|c|}
\hline Scammon (1977) & $\begin{array}{l}\text { Between- } \\
\text { subjects } \\
\text { experimental } \\
\text { design }\end{array}$ & $\begin{array}{l}2 \text { (information } \\
\text { amount: } 4 \text { or } 8 \\
\text { nutrients) } \times 2 \\
\text { (format: \%RDA } \\
\text { or adjectival } \\
\text { descriptions) } \\
\text { design with a } \\
\text { control group }\end{array}$ & $\begin{array}{l}\text { Nutrition labels } \\
\text { embedded in } \\
\text { 30-second } \\
\text { commercials }\end{array}$ & $\begin{array}{l}480 \\
\text { Californians }\end{array}$ & $\begin{array}{l}\text { Accuracy } \\
\text { scores based on } \\
\text { aided recall, } \\
\text { choice quality, } \\
\text { subjective } \\
\text { evaluation of } \\
\text { choice }\end{array}$ & $\begin{array}{l}\text { Increasing } \\
\text { information } \\
\text { (e.g., from } 4 \text { to } \\
8 \text { nutrients } \\
\text { evaluated) } \\
\text { caused } \\
\text { consumers to } \\
\text { divide their } \\
\text { processing time } \\
\text { among the } \\
\text { nutrients, } \\
\text { leading to } \\
\text { information } \\
\text { overload. The } \\
\text { adjectival } \\
\text { format aided } \\
\text { processing, } \\
\text { whereas they } \\
\text { were more } \\
\text { satisfied with } \\
\text { using \%RDAs }\end{array}$ \\
\hline
\end{tabular}




\begin{tabular}{|c|c|c|c|c|c|c|}
\hline Freiden (1981) & $\begin{array}{l}\text { Experimental } \\
\text { design }\end{array}$ & $\begin{array}{l}3 \text { (product } \\
\text { type: wafers, } \\
\text { peaches, } \\
\text { peanut butter) } \\
\times 3 \text { (nutrition } \\
\text { information } \\
\text { amount: low, } \\
\text { medium, high) } \\
\text { design }\end{array}$ & $\begin{array}{l}\text { Vanilla wafers, } \\
\text { sliced peaches, } \\
\text { and peanut } \\
\text { butter } \\
\text { packages }\end{array}$ & $\begin{array}{l}\text { Mall intercepts } \\
\text { with } 135 \\
\text { women in an } \\
\text { Eastern SMSA } \\
\text { city }\end{array}$ & $\begin{array}{l}\text { Attitudes } \\
\text { toward product } \\
\text { stimuli }\end{array}$ & $\begin{array}{l}\text { Brands with } \\
\text { greater } \\
\text { nutrition } \\
\text { information } \\
\text { generated more } \\
\text { favorable } \\
\text { attitudes than } \\
\text { brands with } \\
\text { less } \\
\text { information }\end{array}$ \\
\hline $\begin{array}{l}\text { Brucks et al. } \\
\text { (1984) }\end{array}$ & $\begin{array}{l}\text { Experimental } \\
\text { design }\end{array}$ & $\begin{array}{l}6 \text { (nutrition } \\
\text { format levels: } \\
\text { from none to a } \\
\text { full table with } \\
\text { high quality } \\
\text { values) × } 5 \\
\text { (product type) } \\
\text { design }\end{array}$ & $\begin{array}{l}\text { Fruit juice, } \\
\text { lunchmeat, } \\
\text { dried fruit, } \\
\text { chocolate milk, } \\
\text { French bread } \\
\text { ads }\end{array}$ & $\begin{array}{l}106 \text { married, } \\
\text { middle-class } \\
\text { women }\end{array}$ & $\begin{array}{l}\text { Perceived } \\
\text { nutritiousness, } \\
\text { brand beliefs, } \\
\text { recall, other } \\
\text { beliefs and } \\
\text { purchase } \\
\text { intentions, } \\
\text { nutrition } \\
\text { knowledge }\end{array}$ & $\begin{array}{l}\text { Nutrition } \\
\text { information } \\
\text { provision only } \\
\text { affected early } \\
\text { stages of } \\
\text { processing; } \\
\text { nutrition } \\
\text { knowledge and } \\
\text { product class } \\
\text { affected } \\
\text { acquisition }\end{array}$ \\
\hline
\end{tabular}




\begin{tabular}{|c|c|c|c|c|c|c|}
\hline Muller (1985) & $\begin{array}{l}\text { In-store field } \\
\text { experiment } \\
\text { with control }\end{array}$ & $\begin{array}{l}4 \text { (structural } \\
\text { information } \\
\text { factor } \\
\text { information: } \\
\text { format- } \\
\text { present/not } \\
\text { present, brand } \\
\text { variation, } \\
\text { nutrient } \\
\text { importance, } \\
\text { information } \\
\text { amount) levels }\end{array}$ & $\begin{array}{l}\text { Point-of- } \\
\text { purchase } \\
\text { nutrition signs }\end{array}$ & 138 adults & $\begin{array}{l}\text { Changes in } \\
\text { brand sales }\end{array}$ & $\begin{array}{l}\text { Changes in } \\
\text { brand sales } \\
\text { were a function } \\
\text { of information } \\
\text { format and } \\
\text { variation in } \\
\text { brand ratings } \\
\text { on the sign }\end{array}$ \\
\hline $\begin{array}{l}\text { Russo et al. } \\
(1986)\end{array}$ & $\begin{array}{l}\text { Two field study } \\
\text { experiments }\end{array}$ & $\begin{array}{l}5 \text { information } \\
\text { formats: } \\
\text { matrix, } \\
\text { summary, } \\
\text { complete, } \\
\text { intermediate, } \\
\text { control; neutral } \\
\text { versus negative } \\
\text { information } \\
\text { (added sugar) }\end{array}$ & $\begin{array}{l}\text { Lists of } \\
\text { nutrition } \\
\text { information } \\
\text { posted in } \\
\text { supermarkets }\end{array}$ & 3,254 shoppers & $\begin{array}{l}\text { Nutrition } \\
\text { knowledge, } \\
\text { sales and } \\
\text { market share }\end{array}$ & $\begin{array}{l}\text { Lists of } \\
\text { vitamins/ } \\
\text { minerals } \\
\text { increased } \\
\text { nutrition } \\
\text { knowledge: } \\
\text { added sugar } \\
\text { disclosure } \\
\text { increased } \\
\text { market share of } \\
\text { low-sugar } \\
\text { products }\end{array}$ \\
\hline
\end{tabular}




\begin{tabular}{|c|c|c|c|c|c|c|}
\hline $\begin{array}{l}\text { Venkatesan et } \\
\text { al. (1986) }\end{array}$ & $\begin{array}{l}\text { Repeated } \\
\text { measures } \\
\text { experiment }\end{array}$ & $\begin{array}{l}3 \text { (format: RDA } \\
\text { Index, } \\
\text { Nutritional } \\
\text { Index, } \\
\text { Nutrition } \\
\text { Scoreboard) × } \\
3 \text { (product: } \\
\text { vegetable, } \\
\text { juice, dessert) } \\
\times 2 \text { (media: TV, } \\
\text { print) design }\end{array}$ & $\begin{array}{l}\text { Nutrition label } \\
\text { formats in } \\
\text { television and } \\
\text { print media }\end{array}$ & $\begin{array}{l}75 \text { adults from } \\
\text { a purchasing } \\
\text { panel }\end{array}$ & $\begin{array}{l}\text { Consumer } \\
\text { processing and } \\
\text { policy } \\
\text { measures }\end{array}$ & $\begin{array}{l}\text { RDA format } \\
\text { was preferred } \\
\text { for print } \\
\text { condition, } \\
\text { whereas it was } \\
\text { not for the TV } \\
\text { condition }\end{array}$ \\
\hline Moorman (1990) & $\begin{array}{l}\text { Between- } \\
\text { subjects } \\
\text { experiment }\end{array}$ & $\begin{array}{l}3 \text { (consequence } \\
\text { information: } \\
\text { high, low, } \\
\text { control) × } 2 \\
\text { (reference } \\
\text { information: } \\
\text { present, } \\
\text { absent) × } 2 \\
\text { (nutrient } \\
\text { familiarity: } \\
\text { familiar, } \\
\text { unfamiliar) } \\
\text { design }\end{array}$ & $\begin{array}{l}\text { Shopping list } \\
\text { and } \\
\text { information } \\
\text { sheet for hot } \\
\text { dogs, } \\
\text { margarine }\end{array}$ & $\begin{array}{l}274 \text { staff at a } \\
\text { northeastern } \\
\text { university }\end{array}$ & $\begin{array}{l}\text { Information } \\
\text { processing and } \\
\text { decision quality }\end{array}$ & $\begin{array}{l}\text { Arousing and } \\
\text { specific } \\
\text { consequence } \\
\text { information } \\
\text { aided } \\
\text { processing, } \\
\text { elaboration, } \\
\text { and choices. } \\
\text { Reference } \\
\text { information } \\
\text { affected only } \\
\text { comprehension }\end{array}$ \\
\hline
\end{tabular}




\begin{tabular}{|c|c|c|c|c|c|c|}
\hline $\begin{array}{l}\text { Burton et al. } \\
\text { (1994) }\end{array}$ & $\begin{array}{l}\text { Between- } \\
\text { subjects } \\
\text { experiment }\end{array}$ & $\begin{array}{l}4 \text { (label format) } \\
\times 3 \text { (reference } \\
\text { value) } \times 2 \\
\text { (nutritional } \\
\text { value) design; } \\
\text { nutrition } \\
\text { knowledge } \\
\text { measured }\end{array}$ & $\begin{array}{l}\text { Nutrition } \\
\text { information for } \\
\text { frozen dinners }\end{array}$ & $\begin{array}{l}500 \text { primary } \\
\text { food shoppers }\end{array}$ & $\begin{array}{l}\text { Beliefs, } \\
\text { attitudes, } \\
\text { purchase } \\
\text { likelihood, } \\
\text { accuracy, } \\
\text { understandabili } \\
\text { ty }\end{array}$ & $\begin{array}{l}\text { Inclusion of } \\
\text { reference } \\
\text { amounts and } \\
\text { higher nutrition } \\
\text { knowledge } \\
\text { resulted in } \\
\text { greater } \\
\text { purchase } \\
\text { likelihood for } \\
\text { nutritious } \\
\text { product }\end{array}$ \\
\hline $\begin{array}{l}\text { Viswanathan } \\
\text { (1994) }\end{array}$ & Experiment & $\begin{array}{l}2 \text { (relative } \\
\text { brand } \\
\text { healthiness: } \\
\text { healthy, } \\
\text { unhealthy) × } 4 \\
\text { (reference } \\
\text { format: no- } \\
\text { summary, } \\
\text { average, range, } \\
\text { verbal) design }\end{array}$ & $\begin{array}{l}\text { Nutrition } \\
\text { information for } \\
\text { four brands of } \\
\text { breakfast } \\
\text { cereal }\end{array}$ & $\begin{array}{l}50 \\
\text { undergraduate } \\
\text { students at a } \\
\text { midwestern } \\
\text { university }\end{array}$ & $\begin{array}{l}\text { Healthiness } \\
\text { ratings, recall/ } \\
\text { recognition } \\
\text { accuracy, time } \\
\text { spent on } \\
\text { information }\end{array}$ & $\begin{array}{l}\text { Verbal } \\
\text { presentation of } \\
\text { nutrition } \\
\text { information led } \\
\text { to greater } \\
\text { usage than } \\
\text { numerical } \\
\text { presentations }\end{array}$ \\
\hline
\end{tabular}




\begin{tabular}{|c|c|c|c|c|c|c|}
\hline $\begin{array}{l}\text { Levy et al. } \\
\text { (1996) }\end{array}$ & $\begin{array}{l}\text { Experiment (4 } \\
\times 4 \text { Greco-Latin } \\
\text { square design) }\end{array}$ & $\begin{array}{l}7 \text { format } \\
\text { alternatives } \\
\text { (control, } \\
\text { control/DRV, } \\
\text { adjectival, } \\
\text { grouping, } \\
\text { highlighting, } \\
\text { percent/DRV, } \\
\text { percent) } \\
\text { presented as } \\
\text { part of } 4 \\
\text { different } \\
\text { format-product } \\
\text { combinations }\end{array}$ & $\begin{array}{l}\text { Nutritional } \\
\text { labels for four } \\
\text { different } \\
\text { products }\end{array}$ & $\begin{array}{l}1216 \text { primary } \\
\text { food shoppers } \\
\text { from mall } \\
\text { intercepts }\end{array}$ & $\begin{array}{l}\text { Comparison } \\
\text { and } \\
\text { performance } \\
\text { tasks }\end{array}$ & $\begin{array}{l}\text { Summary } \\
\text { indicators of } \\
\text { nutrient levels } \\
\text { (e.g., } \\
\text { adjectives) and } \\
\text { common } \\
\text { metrics for } \\
\text { interpretation } \\
\text { (e.g., percent } \\
\text { declarations) } \\
\text { had greatest } \\
\text { impact. }\end{array}$ \\
\hline Moorman (1996) & $\begin{array}{l}\text { Longitudinal, } \\
\text { quasi- } \\
\text { experiment }\end{array}$ & $\begin{array}{l}\text { Pre- and post- } \\
\text { NLEA } \\
\text { comparisons }\end{array}$ & $\begin{array}{l}20 \text { different } \\
\text { product } \\
\text { categories }\end{array}$ & $\begin{array}{l}\text { Over } 1,000 \\
\text { consumers } \\
\text { observed and } \\
\text { surveyed in } \\
\text { supermarkets }\end{array}$ & $\begin{array}{l}\text { Nutrition } \\
\text { information } \\
\text { acquisition and } \\
\text { comprehension }\end{array}$ & $\begin{array}{l}\text { Consumers } \\
\text { acquired and } \\
\text { comprehended } \\
\text { more } \\
\text { nutritional } \\
\text { information } \\
\text { following the } \\
\text { introduction of } \\
\text { the new NFPs }\end{array}$ \\
\hline
\end{tabular}




\begin{tabular}{|c|c|c|c|c|c|c|}
\hline $\begin{array}{l}\text { Barone et al. } \\
\text { (1996) }\end{array}$ & $\begin{array}{l}\text { Between- } \\
\text { subjects } \\
\text { experiment }\end{array}$ & $\begin{array}{l}2 \text { (nutrition } \\
\text { value: strong, } \\
\text { weak) } \times 3 \\
\text { (reference } \\
\text { point: none, } \\
\% D V \text {, average } \\
\text { brand) design }\end{array}$ & $\begin{array}{l}\text { Nutritional } \\
\text { labels for } \\
\text { breakfast } \\
\text { cereals }\end{array}$ & $\begin{array}{l}127 \\
\text { undergraduate } \\
\text { students }\end{array}$ & $\begin{array}{l}\text { Overall } \\
\text { healthiness, } \\
\text { nutrition } \\
\text { comprehension, } \\
\text { brand } \\
\text { attitudes, and } \\
\text { intentions }\end{array}$ & $\begin{array}{l}\text { Average brand } \\
\text { values led to } \\
\text { better nutrition } \\
\text { comprehension } \\
\text { than \%DVs }\end{array}$ \\
\hline $\begin{array}{l}\text { Burton and } \\
\text { Andrews (1996) }\end{array}$ & $\begin{array}{l}\text { Between- } \\
\text { subjects } \\
\text { experiment }\end{array}$ & $\begin{array}{l}3 \text { (label format: } \\
\text { full, simplified, } \\
\text { pre-NLEA) } \times 2 \\
\text { (nutrition level: } \\
\text { high, low) } \times 2 \\
\text { (age: under } 58 \text {, } \\
58 \text { or more) } \\
\text { design }\end{array}$ & $\begin{array}{l}\text { Nutrition labels } \\
\text { for frozen } \\
\text { chicken dinners }\end{array}$ & $\begin{array}{l}191 \text { consumer } \\
\text { panel members }\end{array}$ & $\begin{array}{l}\text { Nutrition } \\
\text { evaluation } \\
\text { measures }\end{array}$ & $\begin{array}{l}\text { Older } \\
\text { consumers } \\
\text { displayed } \\
\text { smaller } \\
\text { evaluation } \\
\text { differences for } \\
\text { nutrition levels; } \\
\text { perceived all } \\
\text { labels are more } \\
\text { difficult to } \\
\text { understand }\end{array}$ \\
\hline
\end{tabular}




\begin{tabular}{|c|c|c|c|c|c|c|}
\hline Ford et al. (1996) & $\begin{array}{l}\text { Between- } \\
\text { subjects } \\
\text { experiment }\end{array}$ & $\begin{array}{l}2 \text { (health claim: } \\
\text { present, } \\
\text { absent) × } 2 \\
\text { (NFP } \\
\text { information } \\
\text { favorability; } \\
\text { favorable, } \\
\text { unfavorable) × } \\
2 \text { (ambiguity/ } \\
\text { ease of } \\
\text { interpretation } \\
\text { of nutrition } \\
\text { information: } \\
\text { ambiguous, } \\
\text { unambiguous) } \\
\text { design }\end{array}$ & $\begin{array}{l}\text { Frozen dinner } \\
\text { package }\end{array}$ & $\begin{array}{l}325 \text { business } \\
\text { students }\end{array}$ & $\begin{array}{l}\text { Nutrient beliefs } \\
\text { and overall } \\
\text { healthfulness }\end{array}$ & $\begin{array}{l}\text { Consumers } \\
\text { were accurate } \\
\text { in the } \\
\text { nutritional } \\
\text { evaluation of } \\
\text { products from } \\
\text { the NFP, even } \\
\text { in the presence } \\
\text { of } \\
\text { contradictory } \\
\text { health claims }\end{array}$ \\
\hline
\end{tabular}




\begin{tabular}{|c|c|c|c|c|c|c|}
\hline $\begin{array}{l}\text { Keller et al. } \\
(1997)\end{array}$ & $\begin{array}{l}\text { Between- } \\
\text { subjects } \\
\text { experiment }\end{array}$ & $\begin{array}{l}4 \text { (nutrient } \\
\text { content claim } \\
\text { type) } \times 3 \\
\text { (product } \\
\text { nutrition value) } \\
\times 2 \text { (consumer } \\
\text { motivation to } \\
\text { process } \\
\text { nutrition } \\
\text { information) } \\
\text { design }\end{array}$ & $\begin{array}{l}\text { Frozen chicken } \\
\text { dinner package }\end{array}$ & $\begin{array}{l}800 \text { members } \\
\text { of a statewide } \\
\text { household } \\
\text { research panel }\end{array}$ & $\begin{array}{l}\text { Nutrition and } \\
\text { product } \\
\text { attitudes, } \\
\text { credibility, } \\
\text { purchase } \\
\text { intentions }\end{array}$ & $\begin{array}{l}\text { Motivation } \\
\text { moderated } \\
\text { effects of } \\
\text { nutrition value } \\
\text { on evaluations, } \\
\text { Claims interact } \\
\text { with value à } \\
\text { credibility, but } \\
\text { did not affect } \\
\text { product or } \\
\text { purchase } \\
\text { intentions }\end{array}$ \\
\hline $\begin{array}{l}\text { Szykman et al. } \\
(1997)\end{array}$ & Survey data & $\begin{array}{l}\text { Food Label Use } \\
\text { and Education } \\
\text { Survey } \\
\text { (FLUNES): } \\
\text { random-digit } \\
\text { dialing sample } \\
\text { conducted in } \\
\text { three phases }\end{array}$ & $\begin{array}{l}\text { General } \\
\text { nutritional } \\
\text { questions }\end{array}$ & $\begin{array}{l}\text { 1,812 members } \\
\text { of FLUNES } \\
\text { survey panel }\end{array}$ & $\begin{array}{l}\text { General diet, } \\
\text { disease, } \\
\text { knowledge, } \\
\text { nutritional } \\
\text { label use, } \\
\text { health status, } \\
\text { and other } \\
\text { questions }\end{array}$ & $\begin{array}{l}\text { Diet à disease } \\
\text { perceptions } \\
\text { and knowledge } \\
\text { related to use } \\
\text { of nutrition } \\
\text { panel } \\
\text { information } \\
\text { and package } \\
\text { claims }\end{array}$ \\
\hline
\end{tabular}




\begin{tabular}{|c|c|c|c|c|c|c|}
\hline $\begin{array}{l}\text { Mazis and } \\
\text { Raymond (1997) }\end{array}$ & Experiment & $\begin{array}{l}\text { Manipulation of } \\
\text { information } \\
\text { source for } \\
\text { health claims: } \\
\text { either an ad, } \\
\text { product label, } \\
\text { or product } \\
\text { label with } \\
\text { nutrition } \\
\text { information }\end{array}$ & $\begin{array}{l}\text { Two brands } \\
\text { from each of } \\
\text { the following } \\
\text { presented: } \\
\text { cereal, peanut } \\
\text { butter, soup, } \\
\text { frozen entrees, } \\
\text { and margarine }\end{array}$ & $\begin{array}{l}180 \text { women } \\
\text { primary food } \\
\text { shoppers }\end{array}$ & $\begin{array}{l}\text { Nutrition belief } \\
\text { measures }\end{array}$ & $\begin{array}{l}\text { Only health } \\
\text { claims à } \\
\text { positive effect } \\
\text { on beliefs }\end{array}$ \\
\hline $\begin{array}{l}\text { Mitra et al. } \\
\text { (1999) }\end{array}$ & $\begin{array}{l}\text { Field } \\
\text { experiment }\end{array}$ & $\begin{array}{l}2 \text { (front panel: } \\
\text { implied health } \\
\text { claim, no } \\
\text { health claim) × } \\
4 \text { (back panel: } \\
\text { four different } \\
\text { nutrition levels) } \\
\times 2 \text { (education: } \\
\text { high school } \\
\text { plus, less than } \\
\text { high school) } \\
\text { design }\end{array}$ & $\begin{array}{l}\text { Frozen dinner } \\
\text { package }\end{array}$ & $\begin{array}{l}410 \\
\text { participants } \\
\text { from } 5 \text { cities } \\
\text { interviewed at } \\
\text { home }\end{array}$ & $\begin{array}{l}\text { Nutrient } \\
\text { beliefs, } \\
\text { knowledge, } \\
\text { education }\end{array}$ & $\begin{array}{l}\text { Regardless of } \\
\text { educational } \\
\text { levels, } \\
\text { consumers } \\
\text { were capable of } \\
\text { evaluating and } \\
\text { using the NFP } \\
\text { even in the } \\
\text { presence of a } \\
\text { contradictory } \\
\text { implied health } \\
\text { claim }\end{array}$ \\
\hline
\end{tabular}




\begin{tabular}{|c|c|c|c|c|c|c|}
\hline Roe et al. (1999) & $\begin{array}{l}\text { Experiment } \\
\text { (did not force } \\
\text { exposure to } \\
\text { NFP) }\end{array}$ & $\begin{array}{l}3 \text { (product: } \\
\text { cereal, yogurt, } \\
\text { lasagna) × } 10 \\
\text { (label } \\
\text { condition: } \\
\text { control with no } \\
\text { claim, nutrient } \\
\text { content claim } \\
\text { only, } 8 \text { health } \\
\text { claim formats } \\
\text { with a nutrient } \\
\text { content claim) } \\
\text { design }\end{array}$ & $\begin{array}{l}\text { Cereal, frozen } \\
\text { yogurt, and } \\
\text { lasagna food } \\
\text { packages with } \\
\text { NFPs }\end{array}$ & $\begin{array}{l}1,403 \text { primary } \\
\text { food shoppers } \\
\text { from eight mall } \\
\text { intercepts }\end{array}$ & $\begin{array}{l}\text { Healthiness, } \\
\text { purchase } \\
\text { intent, and } \\
\text { perceived } \\
\text { health benefits }\end{array}$ & $\begin{array}{l}\text { Presence of a } \\
\text { health claim, } \\
\text { and to some } \\
\text { extent a } \\
\text { nutrient } \\
\text { content claim, } \\
\text { significantly } \\
\text { increased the } \\
\text { likelihood that } \\
\text { respondents } \\
\text { truncated their } \\
\text { search to only } \\
\text { the front panel } \\
\text { (and not to the } \\
\text { NFP) }\end{array}$ \\
\hline Li et al. (2000) & Experiment & $\begin{array}{l}2 \text { (nutritional } \\
\text { label value: } \\
\text { higher, lower) } \\
\times 2 \text { (label: \%DV } \\
\text { present, } \\
\text { absent) } 2 \text { (label } \\
\text { knowledge: } \\
\text { higher, lower) } \\
\text { design }\end{array}$ & $\begin{array}{l}\text { Nutritional } \\
\text { label for } \\
\text { cracker brand }\end{array}$ & $\begin{array}{l}205 \\
\text { undergraduate } \\
\text { students }\end{array}$ & $\begin{array}{l}\text { Healthiness, } \\
\text { knowledge, } \\
\text { product } \\
\text { attitude, and } \\
\text { trial intentions }\end{array}$ & $\begin{array}{l}\text { Usefulness of } \\
\text { the \%DVs in } \\
\text { the NFPs } \\
\text { depends on } \\
\text { one's } \\
\text { nutritional } \\
\text { knowledge }\end{array}$ \\
\hline
\end{tabular}




\begin{tabular}{|c|c|c|c|c|c|c|}
\hline $\begin{array}{l}\text { Garretson and } \\
\text { Burton (2000) }\end{array}$ & $\begin{array}{l}\text { Between- } \\
\text { subjects } \\
\text { experiment }\end{array}$ & $\begin{array}{l}3 \text { (NFP } \\
\text { nutritional } \\
\text { value for fat/ } \\
\text { fiber) } \times 5 \\
\text { (claim } \\
\text { information for } \\
\text { fat/fiber) } \\
\text { design }\end{array}$ & $\begin{array}{l}\text { Frozen dinner } \\
\text { package }\end{array}$ & $\begin{array}{l}382 \text { members } \\
\text { of a statewide } \\
\text { consumer panel }\end{array}$ & $\begin{array}{l}\text { Diet-disease } \\
\text { risk, attitudes, } \\
\text { intentions, } \\
\text { credibility, trust }\end{array}$ & $\begin{array}{l}\text { NFP } \\
\text { information on } \\
\text { fat affected } \\
\text { product } \\
\text { evaluations, but } \\
\text { fiber did not. } \\
\text { Claims did not } \\
\text { impact product } \\
\text { evaluations or } \\
\text { intentions }\end{array}$ \\
\hline $\begin{array}{l}\text { Viswanathan } \\
\text { and Hastak } \\
\text { (2002) }\end{array}$ & $\begin{array}{l}\text { Four } \\
\text { experiments }\end{array}$ & $\begin{array}{l}\text { Effects of } \\
\text { summary } \\
\text { information } \\
\text { (average or } \\
\text { range) versus } \\
\text { \%DVs; single } \\
\text { versus multiple } \\
\text { brands } \\
\text { compared }\end{array}$ & $\begin{array}{l}\text { Potato chips } \\
\text { and pretzels }\end{array}$ & $\begin{array}{l}153 \text { student } \\
\text { subjects }\end{array}$ & $\begin{array}{l}\text { Evaluation of } \\
\text { nutritional } \\
\text { content }\end{array}$ & $\begin{array}{l}\text { Summary } \\
\text { information } \\
\text { (average } \\
\text { values) had a } \\
\text { greater effect } \\
\text { on nutritional } \\
\text { evaluations } \\
\text { than \%DVs. } \\
\text { This effect is } \\
\text { reduced when } \\
\text { comparing } \\
\text { multiple brands }\end{array}$ \\
\hline
\end{tabular}




\begin{tabular}{|c|c|c|c|c|c|c|}
\hline $\begin{array}{l}\text { Kozup et al. } \\
(2003)\end{array}$ & $\begin{array}{l}\text { Three } \\
\text { experiments }\end{array}$ & $\begin{array}{l}\text { Effects of } \\
\text { health claims } \\
\text { and nutritional } \\
\text { information } \\
\text { values }\end{array}$ & $\begin{array}{l}\text { Frozen lasagna } \\
\text { dinner and } \\
\text { chicken dinners }\end{array}$ & $\begin{array}{l}147 \text { (study } 1 \text { ) } \\
\text { and } 145 \text { (study } \\
2 \text { ) members of } \\
\text { a consumer } \\
\text { research panel; } \\
364 \text { mall } \\
\text { shoppers (study } \\
\text { 3) }\end{array}$ & $\begin{array}{l}\text { Consumer } \\
\text { evaluations of } \\
\text { disease risk, } \\
\text { product and } \\
\text { nutrition } \\
\text { attitudes, } \\
\text { purchase } \\
\text { intentions, } \\
\text { credibility }\end{array}$ & $\begin{array}{l}\text { Favorable } \\
\text { nutrition } \\
\text { information or } \\
\text { health claims } \\
\text { had } \\
\text { independent } \\
\text { effects on } \\
\text { consumer } \\
\text { evaluations }\end{array}$ \\
\hline $\begin{array}{l}\text { Block and } \\
\text { Peracchio (2006) }\end{array}$ & Three studies & $\begin{array}{l}\text { Effects of } \\
\text { nutrition label } \\
\text { formats (e.g., } \\
\text { simple \%DVs, } \\
\text { \%DVs with } \\
\text { interpretational } \\
\text { aid) on calorie } \\
\text { and calcium } \\
\text { comprehension }\end{array}$ & $\begin{array}{l}\text { Yogurt, whole } \\
\text { milk NFPs }\end{array}$ & $\begin{array}{l}55 \text { Florida } \\
\text { residents } \\
\text { (study 1), } 20 \\
\text { physicians } \\
\text { (study 2), } 41 \\
\text { pregnant or } \\
\text { breast-feeding } \\
\text { women (study } \\
\text { 3) }\end{array}$ & $\begin{array}{l}\text { Comprehension } \\
\text { and usage of } \\
\text { NFPs for } \\
\text { calorie and } \\
\text { calcium levels }\end{array}$ & $\begin{array}{l}\text { Difficulty of } \\
\text { older } \\
\text { consumers and } \\
\text { physicians to } \\
\text { interpret } \\
\text { \%DVs; \%DV } \\
\text { interpretation } \\
\text { aid helped with } \\
\text { usage for } \\
\text { pregnant or } \\
\text { breast-feeding } \\
\text { women }\end{array}$ \\
\hline
\end{tabular}




\begin{tabular}{|c|c|c|c|c|c|c|}
\hline $\begin{array}{l}\text { Wansink and } \\
\text { Chandon (2006) }\end{array}$ & Three studies & $\begin{array}{l}\text { Assessed the } \\
\text { effects of low } \\
\text { fat nutritional } \\
\text { labels on actual } \\
\text { consumption } \\
\text { behavior }\end{array}$ & $\begin{array}{l}\text { M\&Ms and } \\
\text { granola } \\
\text { products }\end{array}$ & $\begin{array}{l}293 \text { university } \\
\text { open house } \\
\text { visitors (study } \\
\text { 1), } 74 \\
\text { university staff } \\
\text { (study 2), } 210 \\
\text { university staff } \\
\text { and students } \\
\text { (study 3) }\end{array}$ & $\begin{array}{l}\text { Actual versus } \\
\text { estimate } \\
\text { calories, } \\
\text { perceived } \\
\text { serving size/ } \\
\text { calorie density, } \\
\text { consumption } \\
\text { guilt }\end{array}$ & $\begin{array}{l}\text { Low-fat } \\
\text { nutritional } \\
\text { labels led all } \\
\text { consumers- } \\
\text { especially those } \\
\text { overweight-to } \\
\text { misinterpret } \\
\text { nutrients/ } \\
\text { serving sizes } \\
\text { and overeat } \\
\text { snack foods }\end{array}$ \\
\hline $\begin{array}{l}\text { Kemp et al. } \\
(2007)\end{array}$ & Experiment & $\begin{array}{l}3 \text { (NFP: low } \\
\text { carb/high fat, } \\
\text { high carb/high } \\
\text { fat, no } \\
\text { nutritional } \\
\text { information) × } \\
3 \text { (claim: low } \\
\text { fat, low carb, } \\
\text { no claim) } \\
\text { design }\end{array}$ & $\begin{array}{l}\text { Frozen dinner } \\
\text { products }\end{array}$ & $\begin{array}{l}270 \text { members } \\
\text { of a household } \\
\text { research panel } \\
\text { mailed packets }\end{array}$ & $\begin{array}{l}\text { Disease } \\
\text { likelihood, } \\
\text { nutrition } \\
\text { perceptions, } \\
\text { purchase } \\
\text { intentions; } \\
\text { motivation }\end{array}$ & $\begin{array}{l}\text { Low-carb } \\
\text { claims } \\
\text { increased } \\
\text { purchase } \\
\text { intentions for } \\
\text { those low in } \\
\text { motivation to } \\
\text { process } \\
\text { nutritional } \\
\text { information }\end{array}$ \\
\hline
\end{tabular}


Nutrition Labeling in the United States and the Role of Consumer Processing, Message Structure, and Moderating Conditions

\begin{tabular}{|c|c|c|c|c|c|c|}
\hline $\begin{array}{l}\text { Viswanathan et } \\
\text { al. (2009) }\end{array}$ & Experiment & $\begin{array}{l}4 \text { (nutritional } \\
\text { label: \%DV, } \\
\text { average, } \\
\text { graphic rating, } \\
\text { graphic range) } \\
\text { × } 3 \text { (literacy } \\
\text { level: low, } \\
\text { medium, high) } \\
\text { design }\end{array}$ & $\begin{array}{l}\text { Nutritional } \\
\text { labels for } \\
\text { potato chips }\end{array}$ & $\begin{array}{l}214 \\
\text { respondents, } \\
\text { including } 120 \\
\text { from adult } \\
\text { education } \\
\text { centers }\end{array}$ & $\begin{array}{l}\text { Healthfulness } \\
\text { and nutrient } \\
\text { ratings }\end{array}$ & $\begin{array}{l}\text { Literacy levels } \\
\text { had a } \\
\text { significant } \\
\text { effect on } \\
\text { understanding } \\
\text { NFPs. Low- } \\
\text { literate } \\
\text { consumers } \\
\text { benefited more } \\
\text { from graphical } \\
\text { (versus non- } \\
\text { graphical) NFP } \\
\text { formats }\end{array}$ \\
\hline
\end{tabular}




\begin{tabular}{|c|c|c|c|c|c|c|}
\hline $\begin{array}{l}\text { Howlett et al. } \\
(2008)\end{array}$ & $\begin{array}{l}\text { Two } \\
\text { experimental } \\
\text { studies }\end{array}$ & $\begin{array}{l}\text { Study 1: } 2 \text { (low } \\
\text { trans-fat claim: } \\
\text { present, } \\
\text { absent) } \times 2 \\
\text { (trans fat level: } \\
\text { high, low) } \times 2 \\
\text { knowledge: } \\
\text { induced, not } \\
\text { induced) } \\
\text { design; Study } \\
2: 3 \text { (trans fat } \\
\text { claims) } \times 2 \\
\text { (trans fat } \\
\text { levels) } \times 2 \\
(\text { motivation) } \times \\
2 \text { (knowledge) } \\
\text { design }\end{array}$ & $\begin{array}{l}\text { Snack cracker } \\
\text { package }\end{array}$ & $\begin{array}{l}153 \text { diabetics } \\
\text { (study 1) and } \\
409 \text { consumers, } \\
\text { both from } \\
\text { online research } \\
\text { panels }\end{array}$ & $\begin{array}{l}\text { Disease risk } \\
\text { perceptions, } \\
\text { nutrient levels }\end{array}$ & $\begin{array}{l}\text { Consumers at } \\
\text { risk for heart } \\
\text { disease } \\
\text { affected by } \\
\text { consumer } \\
\text { knowledge, } \\
\text { trans fat levels, } \\
\text { and motivation } \\
\text { to process } \\
\text { nutrition } \\
\text { information }\end{array}$ \\
\hline $\begin{array}{l}\text { Cook et al. } \\
(2011)\end{array}$ & Survey data & $\begin{array}{l}\text { National Health } \\
\text { and Nutrition } \\
\text { Examination } \\
\text { Survey } \\
\text { (NHANES): } \\
\text { cross-sectional } \\
\text { study }\end{array}$ & $\begin{array}{l}\text { General } \\
\text { nutritional } \\
\text { questions }\end{array}$ & $\begin{array}{l}2,657 \text { members } \\
\text { of NHANES } \\
\text { survey panel } \\
\text { aged } 45 \text { years } \\
\text { or older }\end{array}$ & $\begin{array}{l}\text { Health history, } \\
\text { use of NFPs, } \\
\text { nutrients }\end{array}$ & $\begin{array}{l}\text { Morbidity } \\
\text { condition à } \\
\text { greater use of } \\
\text { NFP (overall) } \\
\text { and condition- } \\
\text { related } \\
\text { nutrients } \\
\text { (specifically) }\end{array}$ \\
\hline
\end{tabular}




\begin{tabular}{|c|c|c|c|c|c|c|}
\hline $\begin{array}{l}\text { Cook et al. } \\
(2013)\end{array}$ & $\begin{array}{l}\text { Two } \\
\text { experiments } \\
\text { (also a pilot } \\
\text { study first } \\
\text { established } \\
\text { internal } \\
\text { reference } \\
\text { points for } \\
\text { ground beef } \\
\text { nutrients) }\end{array}$ & $\begin{array}{l}\text { Study } 1 \text { : } 2 \\
\text { (lean-to-fat } \\
\text { ratio) } \times 2 \\
\text { (attribute } \\
\text { frame) } \times 2 \\
\text { (NFP presence/ } \\
\text { absence) } \\
\text { between- } \\
\text { subjects } \\
\text { design; Study } \\
2: 2 \text { (NFP } \\
\text { absent/present) } \\
\times 2 \text { (nutrition } \\
\text { consciousness } \\
\text { high/low) } \times 4 \\
\text { (lean-to-fat } \\
\text { ratio) mixed } \\
\text { factorial } \\
\text { design, with } \\
\text { lean-to-fat ratio } \\
\text { as a within- } \\
\text { subjects factor }\end{array}$ & $\begin{array}{l}\text { Nutritional } \\
\text { labels for } \\
\text { ground beef }\end{array}$ & $\begin{array}{l}304 \text { adult } \\
\text { consumers } \\
\text { (study } 1 \text { ) and } \\
344 \text { adults } \\
\text { consumers } \\
\text { (study 2), both } \\
\text { from online } \\
\text { research panels }\end{array}$ & $\begin{array}{l}\text { Perceptions of } \\
\text { disease risk, } \\
\text { product } \\
\text { healthfulness, } \\
\text { nutrient } \\
\text { (attribute) } \\
\text { evaluations }\end{array}$ & $\begin{array}{l}\text { With higher } \\
\text { internal } \\
\text { reference } \\
\text { points for } \\
\text { calories, fat, } \\
\text { and saturated } \\
\text { fat in lean } \\
\text { ground beef, a } \\
\text { disclosure of } \\
\text { actual lower } \\
\text { levels tends to } \\
\text { improve } \\
\text { attribute } \\
\text { evaluations }\end{array}$ \\
\hline
\end{tabular}




\begin{tabular}{|c|c|c|c|c|c|c|}
\hline $\begin{array}{l}\text { Nelson et al. } \\
(2014)\end{array}$ & $\begin{array}{l}\text { Eye-tracking } \\
\text { study }\end{array}$ & $\begin{array}{l}\text { NFP viewing } \\
\text { time as a } \\
\text { function of age } \\
\text { and nutrient } \\
\text { density score of } \\
\text { food }\end{array}$ & $\begin{array}{l}\text { Viewed labels } \\
\text { for } 64 \text { foods } \\
\text { across many } \\
\text { product } \\
\text { categories }\end{array}$ & $\begin{array}{l}202 \text { adult } \\
\text { participants in } \\
\text { an eye-tracking } \\
\text { study }\end{array}$ & $\begin{array}{l}\text { NFP } \\
\text { component } \\
\text { viewing time } \\
\text { via eye-tracker; } \\
\text { Nutrient Rich } \\
\text { Food index, } \\
\text { demographics }\end{array}$ & $\begin{array}{l}\text { Those who } \\
\text { spend more } \\
\text { time viewing } \\
\text { NFPs during a } \\
\text { shopping trip } \\
\text { may not be } \\
\text { selecting more } \\
\text { nutritious foods }\end{array}$ \\
\hline $\begin{array}{l}\text { Gonzalez- } \\
\text { Vallejo et al. } \\
(2016)\end{array}$ & Experiment & $\begin{array}{l}\text { Contrasted } \\
\text { current NFP } \\
\text { with revised } \\
\text { (“modified”) } \\
\text { versions } \\
\text { proposed by } \\
\text { FDA }\end{array}$ & $\begin{array}{l}\text { Either } 40 \\
\text { different } \\
\text { cereals or } 40 \\
\text { different } \\
\text { snacks }\end{array}$ & $\begin{array}{l}196 \text { adult, } \\
\text { online } \\
\text { participants }\end{array}$ & $\begin{array}{l}\text { Judgment } \\
\text { consistency } \\
\text { (correspondenc } \\
\text { e between } \\
\text { consumer } \\
\text { judgments and } \\
\text { actual } \\
\text { nutritional } \\
\text { quality via } \\
\text { NuVal score) }\end{array}$ & $\begin{array}{l}\text { The current } \\
\text { NFP is equally } \\
\text { or more } \\
\text { effective in } \\
\text { conveying } \\
\text { nutritional } \\
\text { information } \\
\text { versus revised } \\
\text { ("modified”) } \\
\text { versions based } \\
\text { on FDA } \\
\text { proposed } \\
\text { changes }\end{array}$ \\
\hline
\end{tabular}

Note: $(*)$ This table is based in part on Hieke and Taylor (2012) and provides an overview of selected consumer studies that examine the effects of Nutrition Facts Panel format, wording, and/or moderating conditions on key outcomes. 
Many of the pre-NLEA consumer studies on nutritional labeling focused on format issues (e.g., complexity, type of content, amount of information). As an example, Asam and Bucklin (1973) found that detailed nutrition label information (e.g., each serving contains 0.4 grams of fat) led to significantly better shopper perceptions and purchase preferences than did more ambiguous nutrition label information (e.g., "low in fat"). Yet, in Scammon (1977), increasing the amount of information (e.g., from four to eight nutrients evaluated) caused consumers to divide their processing time among the nutrients, leading to information overload. In contrast to Asam and Bucklin (1973), a more simplified nutrition format (e.g., adjectival versus percentage RDA) aided the identification of the best brand, yet left consumers feeling dissatisfied and desiring greater information. Finally, in another study before the NFPs were implemented, seven nutrition label formats (e.g., previous, voluntary format (control); control/daily reference values (DVs); adjectival; grouping; highlighting; percent/DRV; percent) were tested on five comprehension tasks (Levy, Fein, \& Schucker, 1996). Thus, some formats had interpretational aids (DRV lists, adjectives, grouped nutrients, or bolded/highlighted nutrients). The formats that had the greatest impact were those that provided summary indicators of nutrient levels (e.g., adjectives) and common metrics for interpretation (e.g., percent declarations) because they reduce the cognitive effort required by the comprehension tasks.

Other research compared pre- and post-NLEA changes to the NFPs. For example, Moorman (1996) found in a longitudinal quasi-experiment across 20 different product categories that consumers acquired and comprehended more nutritional information following the introduction of the new NFPs. Post-NLEA research tended to focus on the given NFP format (e.g., Burton, Garretson, \& Velliquette, 1999), yet also examined wording changes (quantitative and qualitative), as well as moderating conditions (see below). For example, in Howlett, Burton, and Kozup (2008), trans fat knowledge and nutritional levels (in the NFPs) interacted to influence perceptions of cardiovascular risk for those with diabetes. Key demographic factors also are found to influence the understanding of the NFPs, with older consumers having greater difficulty in understanding nutritional levels differences in the NFPs (Burton \& Andrews, 1996) and in using the DV\% in the NFPs (Block \& Peracchio, 2006).

\section{NFP Moderating Conditions}

Perhaps the most important contribution from the consumer research studies on the NFPs involves the consideration of key moderating conditions, such as consumer motivation and ability and opportunity to process nutrition labeling information (see Petty \& Cacioppo, 1986, in general). As aptly noted in their review of nutritional labeling, Derby and Levy (2001, p. 387) caution that “. . information alone will not change behavior." For instance, consumers with higher enduring motivation to process nutrition information were significantly better able to evaluate differences in nutritional value on the NFPs than those lower in motivation (Keller et al., 1997). Consumers with a specific morbidity 
Nutrition Labeling in the United States and the Role of Consumer Processing, Message Structure, and Moderating Conditions

status (e.g., hypertension and/or high cholesterol), who should be motivated to utilize the NFP regarding nutrients closely linked to their condition (e.g., sodium and cholesterol), also were shown to be the most frequent users of calorie and nutrient information included on the NFP (Cook, Burton, \& Howlett, 2011; Howlett, Burton, Tangari, \& Bui, 2012). Yet, in the case of other specific nutrients (e.g., trans fats), motivation in the absence of knowledge can sometimes lead to a misinterpretation of nutrition information (Howlett et al., 2008). As an example, Li, Miniard, and Barone (2000) find that the usefulness of the \%DVs in the NFPs depends on one's nutritional knowledge. Similarly, Viswanathan, Hastak, and Gau (2009) show that consumer literacy levels significantly affect the understanding of the NFPs, and graphical (versus non-graphical) formats aid usage of nutritional information by lower literate consumers. A final moderating condition that is often overlooked is the prior nutritional information (or biases) that consumers bring with them in processing nutritional labels. For instance, Cook, Burton, and Howlett (2013) find that because of higher internal reference points of consumers for calories, fat, and saturated fat in lean ground beef, a disclosure of actual lower levels tends to improve attribute evaluations.

Providing the NFP also influenced disease risk perceptions, an outcome that extends beyond product evaluations, such that less healthful products (e.g., ground beef with 30\% fat content) were perceived as riskier and less likely to be purchased. Results were consistent with previous research on consumers' negativity biases and the unequally weighted effect of negative (more than positive) information on risk considerations (Baumeister, Bratslavsky, Finkenauer, \& Vohs, 2001; Tversky \& Kahneman, 1981). Overall, the presence (versus absence) of the NFP increased the choice of leaner options (e.g., ground beef with only $3 \%$ or $10 \%$ fat content) while decreasing perceptions of healthfulness for fattier options.

\section{Nutrition Facts for Meat and Poultry Products}

A recent update for the provision of the NFP occurred in 2012 when the U.S. Department of Agriculture (USDA) required the NFP for single-ingredient, ground/chopped meat (e.g., ground beef) and poultry products (e.g., turkey). Nutrition information on the package or in the form of point-of-purchase materials also is required for major cuts of meat and poultry (e.g., chicken tenderloin). The new regulations were intended to address the lack of participation by most meat retailers and producers with the voluntary nutrition labeling guidelines established by the USDA nearly two decades earlier in 1993 (Federal Register, 2010). The changes also addressed how lean-to-fat ratio claims (e.g., percent lean only (95\% lean) vs. percent lean/percent fat (95\% lean/5\% fat) could be presented to consumers. Statements of lean percentage that fail the "low fat" regulatory criteria also are prohibited (Federal Register, 2010, p. 82157). 
Nutrition Labeling in the United States and the Role of Consumer Processing, Message Structure, and Moderating Conditions

Relative to the rest of the world, the United States has the largest consumption (in volume) of meat and poultry where most of the products consumed (>75\%) are not processed (i.e., raw; Daniel, Cross, Koebnick, \& Sinha, 2011). In response, the retail equivalent value in 2015 of the U.S. beef industry was \$105 billion and over \$48 billion for poultry sales (USDA, 2016A, 2016B). However, meat may contain large amounts of negative nutrients (e.g., total fat, cholesterol) that epidemiologic evidence has shown can lead to greater cancer and chronic disease risk (CHIUVE ET AL., 2012). Disclosing nutrition information in the meat department now allows consumers to select in an objective manner more healthful products that fit their individual dietary needs.

Several studies have examined how the availability of nutrition information may influence consumers' behavior and choices for products currently covered (e.g., beef, poultry) and not yet covered (e.g., raw seafood) in the legislation. Burton, Cook, Howlett, and Newman, (2015) show how nutrition information helps consumers correct misperceptions about a product's health profile. Blanket assumptions about beef (e.g., "red meat is bad for you"-a health "horn") and poultry (e.g., "white meat is good for you"-a health "halo") are not always consistent with the calorie and nutrient content of products in these categories. For example, sirloin steak has a more favorable nutrition profile (i.e., fewer calories, lower fat content) than whole cut-up chicken with skin. When nutrition information (on the product or on point-of-purchase materials) disconfirms consumers' expectations about healthfulness (e.g., "this product is better (worse) than I expected!"), the effects on perceptions and behavior are more favorable for the healthier beef and poultry choices. Even though nutrition information is not yet required for fresh (raw) seafood, its provision was shown to influence consumers' willingness to pay higher prices (Bi, House, \& Gao, 2016). While research in this domain is still limited, results are largely supportive of the changing information environment. Consumers who utilize nutrition information in the meat department are able to make healthier choices and, when compared to products without a NFP, may be willing to pay more.

\section{Revised NFPs and Opportunities for Research}

On May 20, 2016, the FDA announced that a revised NFP would be introduced that provided a declaration of grams and \%DVs for "added sugars" for the first time and updated design to highlight "calories" and "servings," updated requirements for serving sizes, and "dual column" labels for both "per serving" and "per package" in the case of multi-serving food products, among other changes (FDA, 2016A). This revised NFP ("new label") targeted to go into effect in the near future appears in Figure 1 alongside the original that was implemented in 1994. 


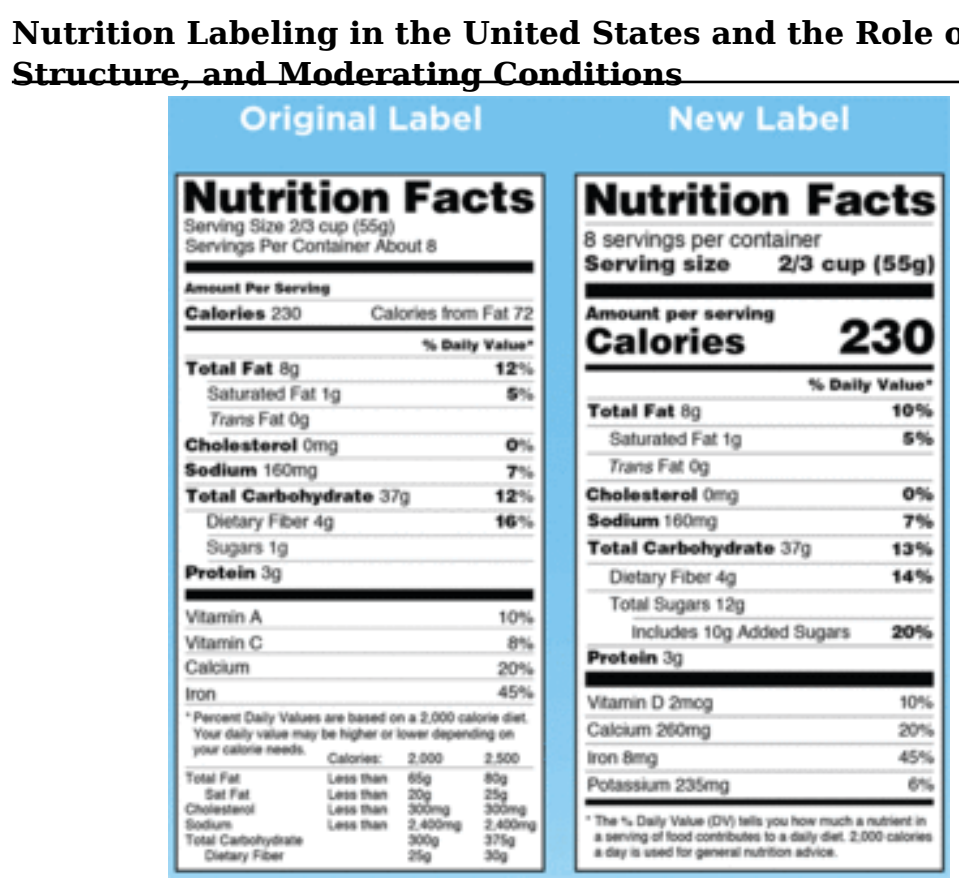

Click to view larger

Figure 1. Original versus new nutrition facts panels.

(Source: FDA, 2016A).
Several studies have examined the revised NFP ("new label") using simple surveys, eye-tracking research, and betweensubjects experimental designs. In Laquatra, Sollid, Edge, Pelzel, and Turner (2015), over 1,000 consumers were probed as to the meaning of "added sugars" on three different versions of a proposed version of the facts panel. The findings indicated that many incorrectly interpreted "added sugars" to mean those in addition to total sugars or

sugars, so the revised label appearing in Figure 1 clarifies that "total sugars" includes "added sugars." In an eye-tracking study by Graham and Roberto (2016), the revised ("modified") NFPs did not elicit significantly more visual attention or lead to more healthful purchase intentions than the current NFP. In fact, one proposed (yet not implemented) change to move the \%DVs to the left of the panel from the right actually reduced consumer attention to this information. In addition, a between-subjects experiment indicated that the current NFP is equally or more effective in conveying nutritional information compared to revised ("modified") versions based on FDA-proposed changes (Gonzalez-Vallejo \& Lavins, 2015). Finally, the FDA (2016B) has conducted eyetracking studies, experiments on alternative formats, and studies on the added sugars and footnote provisions for the revised NFPs, with findings generally consistent with the revised NFP research noted above.

Clearly, further experimental work on revised (new) NFPs is warranted, with the consideration of important moderators, such as nutrition knowledge, motivation, and ability to process the revised NFPs. Ability to process refers to the degree to which consumers have sufficient time and are free from distractions in processing the NFPs. Comprehension tests and choice tasks are needed to compare the revised and currently used NFP, especially regarding the more prominent calorie and added sugars additions. In addition, how revised serving sizes for certain categories affect product evaluations and consumption amounts will be of substantial interest. There also may be unintended consequences of the revised NFPs, with non-caloric artificial sweeteners used more frequently, as noted by Malik, Willett, and Hu (2016). Finally, as the NFP is only one piece 


\section{Calorie Labeling for Restaurant Chains}

As noted previously, the NLEA (1990) exempted restaurants and other businesses selling ready-to-consume prepared foods from mandatory nutrition labeling. However, given the increases in obesity and increased number of calories consumed outside the home, the Institute of Medicine recommended menu calorie labeling in 2005 as a strategy to counter mounting obesity levels (Long, Tobias, Cradock, Batchelder, \& Gortmaker, 2015). By that time, consumers' away-from-home consumption had reached some one-third of their total calories and almost one-half of their food budget. By 2009, several states and a number of cities and counties around the United States, including New York City and King County, Washington, had passed and implemented menu calorie labeling legislation (Roberto, Schwartz, \& Brownell, 2009), and they were followed by California. The states and localities that passed labeling laws from 2007 to 2009 had created differing labeling requirements for menu disclosures. Faced with the operational difficulties of meeting these mandates, which differed in information required and the specific presentation format, the National Restaurant Association and many major restaurant chains supported national legislation that would standardize labeling requirements.

In 2010, a provision in the U.S. Patient Protection and Affordable Care Act (2010) required chain restaurants with 20 or more stores nationally to include calorie information on menus and menu boards. Other nutrition information has to be made available upon request. However, given residual opposition from some business groupsconvenience stores, supermarkets, and take-out-only pizza chains lobbied for exemptions -the FDA struggled to develop its final rules and regulations. Final rules were completed and published in May 2016, and they will require the calorie information on menus and menu boards to be disclosed by May 2017. The final rules will apply to ready-to-eat foods at the following types of outlets:

- Fast food and table service restaurant chains

- Bakeries

- Cafeterias

- Coffee shops

- Convenience stores

- Food takeout and delivery operations (e.g., pizza takeout)

- Delicatessens

- Food concession stands located within entertainment venues (e.g., amusement parks, bowling alleys, and movie theaters) 
- Food service vendors (e.g., ice cream shops, mall cookie counters)

- Grocery stores

- Retail confectionary stores

As noted above, in addition to disclosed calorie levels, for each of these types of retail outlets, nutrient content information for standard items must be available upon request.

\section{Findings from the Literature and Opportunities for Research}

There have been a number of field and lab-based experimental studies conducted since calorie labeling for restaurant chains became operational in state and local governments that previously mandated labeling in restaurants. In general, this research has raised concerns that market-based change to the information environment in restaurant chains will not have widespread effects on consumer choice behavior (e.g., Elbel, Kersh, Brescoll, \& Dixon, 2009; Ellison, Lusk, \& Davis, 2013; Finkelstein, Strombotne, Chan, \& Krieger, 2011; Long et al., 2015; Tandon et al., 2011). A recent meta-analysis of the studies indicated that calorie provision mandates have not significantly decreased calories in meals ordered from restaurants (Long et al., 2015). An overview of these studies is provided in Table 2. Based on the results of the meta-analysis, it was concluded that "there is minimal evidence to support menu calorie labeling as a strategy to directly influence consumer behavior to substantially reduce calories purchased at restaurants" (Long et al., 2015, pp. E21-E22). 
Table 2 Overview of Studies Examining Effects of Calorie Labeling on Calories Ordered in Restaurant Meal Choices

\begin{tabular}{|c|c|c|c|c|c|c|}
\hline $\begin{array}{l}\text { Authors/Date } \\
\text { of Study }\end{array}$ & Study Design & $\begin{array}{l}\text { Manipulations } \\
\text { and Control } \\
\text { Group }\end{array}$ & $\begin{array}{l}\text { Type of } \\
\text { Restaurant }\end{array}$ & Study Sample & $\begin{array}{l}\text { Dependent } \\
\text { Variable }\end{array}$ & $\begin{array}{l}\text { Study } \\
\text { Findings }\end{array}$ \\
\hline $\begin{array}{l}\text { Elbel et al. } \\
\text { (2009) }\end{array}$ & $\begin{array}{l}\text { Longitudinal } \\
\text { experiment } \\
\text { with control }\end{array}$ & $\begin{array}{l}\text { Provision of } \\
\text { menu calorie } \\
\text { labeling in } \\
\text { NYC; no } \\
\text { labeling in a } \\
\text { control city }\end{array}$ & $\begin{array}{l}\text { Fast-food } \\
\text { restaurants }\end{array}$ & $\begin{array}{l}1,125 \text { adult } \\
\text { consumers }\end{array}$ & $\begin{array}{l}\text { Calories } \\
\text { ordered per } \\
\text { meal }\end{array}$ & $\begin{array}{l}\text { Calorie labeling } \\
\text { did not affect } \\
\text { calories } \\
\text { ordered }\end{array}$ \\
\hline $\begin{array}{l}\text { Dumanovsky et } \\
\text { al. (2011) }\end{array}$ & $\begin{array}{l}\text { Longitudinal } \\
\text { experiment } \\
\text { without control }\end{array}$ & $\begin{array}{l}\text { Provision of } \\
\text { menu calorie } \\
\text { labeling in NYC }\end{array}$ & $\begin{array}{l}\text { Fast-food } \\
\text { restaurants }\end{array}$ & $\begin{array}{l}15,798 \text { adult } \\
\text { consumers }\end{array}$ & $\begin{array}{l}\text { Calories } \\
\text { ordered per } \\
\text { meal }\end{array}$ & $\begin{array}{l}\text { Nonsignificant } \\
\text { increase in } \\
\text { calories } \\
\text { ordered }\end{array}$ \\
\hline
\end{tabular}




\begin{tabular}{|c|c|c|c|c|c|c|}
\hline $\begin{array}{l}\text { Finkelstein et } \\
\text { al. (2011) }\end{array}$ & $\begin{array}{l}\text { Longitudinal } \\
\text { experiment } \\
\text { with control }\end{array}$ & $\begin{array}{l}\text { Provision of } \\
\text { menu calorie } \\
\text { labeling in King } \\
\text { County, WA; no } \\
\text { calorie labeling } \\
\text { in control } \\
\text { county }\end{array}$ & $\begin{array}{l}\text { Fast-food } \\
\text { restaurants }\end{array}$ & $\begin{array}{l}\text { Transaction } \\
\text { data from } \\
\text { stores }\end{array}$ & $\begin{array}{l}\text { Calories per } \\
\text { transaction }\end{array}$ & $\begin{array}{l}\text { Calorie labeling } \\
\text { did not affect } \\
\text { calories } \\
\text { ordered }\end{array}$ \\
\hline $\begin{array}{l}\text { Bollinger et al. } \\
(2011)\end{array}$ & $\begin{array}{l}\text { Longitudinal } \\
\text { experiment } \\
\text { with control }\end{array}$ & $\begin{array}{l}\text { Provision of } \\
\text { menu calorie } \\
\text { labeling in } \\
\text { NYC; no } \\
\text { labeling in } \\
\text { Boston and } \\
\text { Philadelphia }\end{array}$ & $\begin{array}{l}\text { Large coffee } \\
\text { shop chain }\end{array}$ & $\begin{array}{l}\text { Starbucks } \\
\text { transaction } \\
\text { data }\end{array}$ & $\begin{array}{l}\text { Calories per } \\
\text { transaction }\end{array}$ & $\begin{array}{l}\text { Minor but } \\
\text { significant } \\
\text { decrease } \\
(-14.4) \text { in } \\
\text { calories per } \\
\text { transaction }\end{array}$ \\
\hline $\begin{array}{l}\text { Tandon et al. } \\
\text { (2011) }\end{array}$ & $\begin{array}{l}\text { Longitudinal } \\
\text { experiment } \\
\text { without control }\end{array}$ & $\begin{array}{l}\text { Provision of } \\
\text { menu calorie } \\
\text { labeling in King } \\
\text { County, WA; no } \\
\text { calorie labeling } \\
\text { in control } \\
\text { county }\end{array}$ & $\begin{array}{l}\text { Fast-food } \\
\text { restaurants }\end{array}$ & $\begin{array}{l}133 \text { pairs of } \\
\text { parents and } \\
\text { children }\end{array}$ & $\begin{array}{l}\text { Calories } \\
\text { ordered per } \\
\text { meal }\end{array}$ & $\begin{array}{l}\text { Calorie labeling } \\
\text { did not affect } \\
\text { calories } \\
\text { ordered }\end{array}$ \\
\hline
\end{tabular}




\begin{tabular}{|c|c|c|c|c|c|c|}
\hline $\begin{array}{l}\text { Ellison et al. } \\
(2013)\end{array}$ & $\begin{array}{l}\text { Experiment } \\
\text { with control }\end{array}$ & $\begin{array}{l}\text { Participants } \\
\text { randomly } \\
\text { assigned to } \\
\text { order from } \\
\text { restaurant } \\
\text { menu with } \\
\text { calorie } \\
\text { labeling, } \\
\text { labeling plus } \\
\text { traffic light, or } \\
\text { no labeling }\end{array}$ & $\begin{array}{l}\text { Sit-down } \\
\text { university } \\
\text { restaurant }\end{array}$ & 138 adults & $\begin{array}{l}\text { Calories } \\
\text { ordered per } \\
\text { meal }\end{array}$ & $\begin{array}{l}\text { Nonsignificant } \\
\text { increase in } \\
\text { calories } \\
\text { ordered }\end{array}$ \\
\hline $\begin{array}{l}\text { Downs et al. } \\
(2013)\end{array}$ & $\begin{array}{l}\text { Longitudinal } \\
\text { experiment } \\
\text { without control }\end{array}$ & $\begin{array}{l}\text { Provision of } \\
\text { menu calorie } \\
\text { labeling in } \\
\text { NYC; } \\
\text { participants } \\
\text { randomly } \\
\text { assigned to } \\
\text { receive per } \\
\text { meal anchor, } \\
\text { daily anchor, or } \\
\text { no anchor }\end{array}$ & $\begin{array}{l}\text { Fast-food } \\
\text { restaurants }\end{array}$ & 1,094 adults & $\begin{array}{l}\text { Calories } \\
\text { ordered per } \\
\text { meal }\end{array}$ & $\begin{array}{l}\text { Nonsignificant } \\
\text { increase in } \\
\text { calories } \\
\text { ordered }\end{array}$ \\
\hline
\end{tabular}




\begin{tabular}{|c|c|c|c|c|c|c|}
\hline $\begin{array}{l}\text { Krieger et al. } \\
\text { (2013) }\end{array}$ & $\begin{array}{l}\text { Longitudinal } \\
\text { experiment } \\
\text { with control }\end{array}$ & $\begin{array}{l}\text { Provision of } \\
\text { menu calorie } \\
\text { labeling in King } \\
\text { County, WA }\end{array}$ & $\begin{array}{l}\text { Fast-food } \\
\text { restaurants and } \\
\text { coffee chain }\end{array}$ & $\begin{array}{l}7,235 \\
\text { participants } \\
\text { over the age of } \\
13\end{array}$ & $\begin{array}{l}\text { Calories } \\
\text { ordered per } \\
\text { meal }\end{array}$ & $\begin{array}{l}\text { Coffee chain: } \\
\text { significant } \\
\text { decrease in } \\
\text { calories } \\
\text { ordered; fast- } \\
\text { food } \\
\text { restaurant: } \\
\text { effect was } \\
\text { nonsignificant }\end{array}$ \\
\hline
\end{tabular}

Note: $(*)$ This table was adapted from Long et al. (2015) and provides an overview of the studies conducted that examine the effect of menu calorie provision on calories ordered from chain restaurants. Based on the six controlled studies in the table, Long et al. (2015) found that calorie provision resulted in an overall nonsignificant decrease in calories ordered (i.e., -7.63 calories). 


\section{Nutrition Labeling in the United States and the Role of Consumer Processing, Message Structure, and Moderating Conditions}

Generally, the strongest, controlled field experiments have used pre-post designs with some type of control group (although randomization is not feasible in most cases). For example, calories per meal were compared in a natural experiment before and after menu calorie labeling was introduced in New York City to calories from a control location (Newark, New Jersey) without labeling (Elbel et al., 2009). Calorie labeling did not influence the mean number of calories ordered by consumers (Elbel et al., 2009). In one of the few studies to show significant decreases, Bollinger, Leslie, and Sorensen (2011) reported a mean decrease of 14.4 calories for an extremely large data set consisting of transaction data obtained from Starbucks.

Such results from field studies are in contrast to many of the findings from experimental menu-based studies conducted in laboratory environments. For example, these lab results have shown that for items that are substantially higher in calories than consumers expect, when exposed to disconfirming calorie information, choices are affected; yet, choices and purchase intentions are unaffected when the calories disclosed are similar to consumers' pre-exposure expectations (e.g., Burton, Creyer, Kees, \& Huggins, 2006; Burton, Howlett, \& Tangari, 2009). Similarly, calorie disclosures have been shown to have a significant effect on consumers high in health/calorie consciousness and motivation to process nutrition information, but little or no effect on those with lower health consciousness (Bates, Burton, Howlett, \& Huggins, 2009; Howlett, Burton, Bates, \& Huggins, 2009). When considering the conceptual foundation of when calorie information disclosures are most likely to have substantial effects, differences in these lab studies focusing on moderating effects versus field studies more interested in the direct effect of including calorie information seem understandable (e.g., Burton \& Kees, 2012). For example, numerous environmental, contextual, and enduring individual-level factors suggest when calorie disclosures will affect choices and calories ordered (Kees, Burton, \& Andrews, 2015; Wansink \& Chandon, 2014). In addition, some of these underlying differences may suggest unintended consequences where disclosures lead to increases in calories ordered (Burton \& Kees, 2012). For example, if consumers are not health conscious, but infer that higher calories suggest superior taste (Raghunathan, Naylor, \& Hoyer, 2006), this may lead to increases in calorie ordered.

As suggested in the model of Burton and Kees (2012) and Kees et al. (2015), there are a number of specific conditions that must be satisfied for calorie labeling to have a favorable impact on the meal order of any specific consumer. These stages include initial awareness, processing conditions, integration and evaluation, and contextual and individual difference variables. Many consumers are frequent repeat customers to restaurants and may habitually order meals based on prior experiences with little attention to menu options or a menu board. In addition, acquisition of information in many menu board or drive-thru venues may be difficult for consumers due to the presentation and size of the calorie information, coupled with the amount of competing information to be processed. This information awareness constraint may initially reduce the size of the segment influenced by a considerable percentage. When the calorie 


\section{Nutrition Labeling in the United States and the Role of Consumer Processing, Message Structure, and Moderating Conditions}

information for competing items is accessed, the level of knowledge and motivation to process the information (Petty \& Cacioppo, 1986) is a critical factor. Findings show that without sufficient motivation there is no effect of labeling, and it has been argued that for consumers strongly valuing taste or quantity, low calories may act as cue that leads to increases in higher-calorie items and meals. Given that attributes such as taste, price, and meal size and satiation are extremely influential in most consumer decisions, it seems intuitive that in many instances objective or inferred levels of these attributes will trump perceived healthfulness.

Past laboratory research also shows that for chain restaurant calorie disclosures to have the desired effect to motivate more healthful choices, the information should provide "new" information that indicates that previously held beliefs about the relative calorie level of the item have been inaccurate (Burton et al., 2006). If calorie information merely confirms prior expectations, then little change in choice behavior is anticipated. In addition, there are many environmental and situational factors that may influence or bias consumer processing (Chandon \& Wansink, 2007; Wansink \& Chandon, 2014). Such environmental and contextual influences may far outweigh the effects of objective calorie information.

Beyond these effects, which indicate many of the impediments to effectiveness, inferences about taste and meal size may be directly affected by higher calorie content (e.g., Raghunathan, Naylor, \& Hoyer, 2006). Given the diverse consumer differences in food choice motivations (maximization of taste, quantity/value, convenience, and emotional comfort), there are many consumers for which calorie labeling is very unlikely to have intended effects and, at times, may increase calorie consumption.

Because calorie labeling is most likely to have a substantial favorable effect on a rather select segment, at least initially, several directions for future research directions are indicated. When and how disclosures are effective suggests possible, very complex higher-order interactions (e.g., calorie expectations $\times$ consumer motivation $\times$ situational context) that may be addressed. Which of these factors is most influential in overriding a high level of consumer motivation to choose healthier options? Can educational campaigns or "nudges" at the point of purchase help to influence not only the more motivated, health-conscious consumers, but also the segments less likely to be influenced by calorie disclosures? What is the effect of labeling on nutrient consumption from restaurant fare, such as sodium, that is very high and substantially underestimated but not included in the labeling mandate (Burton, Tangari, Howlett, \& Turri, 2014; Howlett et al., 2012)? How will restaurant management respond in terms of their offers of product portfolio mixes, and can presentation formats (grouping of more healthful/less healthful options) affect consumer processing and fluency of the processing of a large menu of options, ultimate choices, and satisfaction with the restaurant? Similarly, how will the level of calorie content relative to other critical evaluation attributes (price, perceived taste, size) and time pressure interact to affect choices in their effects (Parker \& Lehmann, 2014)? And, given that the legislation requires disclosures for a broad set of institutions, including retailers that offer prepared food at grocery stores, convenience 
Nutrition Labeling in the United States and the Role of Consumer Processing, Message Structure, and Moderating Conditions

stores, take-out-only chains, movie theaters, bakeries, cafeterias, and coffee shops (and others), what will be the relative effect of labeling across these different venues covered by the final rules of the FDA? While most of the field-based studies have focused directly on specific calorie consumption effects for diners (Long et al., 2015), when calorie disclosures are implemented in May 2017, future studies should address more complex interaction-moderating effects (Burton \& Kees, 2012) and longer-term consequences for diverse groups of consumers and different types of retailer firms.

\section{Front-of-Pack Nutrition Labeling Systems}

\section{Brief History of FOP Labeling and Systems}

Since approximately 2006, U.S. consumers have been exposed to numerous front-ofpackage (FOP) nutrition symbols and icons, including the "Smart Choices" icon, Kellogg's and Mars' use of the Guideline Daily Amounts, Hannaford's Guiding Stars, the AHA's Heart-Check mark, Wal-Mart's "Great for You” Program, the Grocery Manufacturers of American (GMA) and Food Marketing Institute (FMI)'s Facts Up Front (FUF) system, the NuVal Scoring System, and the Institute of Medicine's proposed system and symbol (AHA, 2014; CSPI, 2006; GMA, 2011; GMA \& FMI, 2011; IOM, 2011; NuVal, 2014; Sebolt, 2008). Perhaps the best way to categorize the many FOP symbols is as either (1) a summary or evaluative symbol (e.g., Smart Choices, IOM, NuVal) in providing consumers with an overall evaluation of a product's healthfulness or (2) a nutrient-specific or reductive symbol (e.g., U.K.'s traffic lights; FUF) that presents a reduced amount or "snapshot" of information from the nutrition facts label (Andrews, Lin, Levy, \& Lo, 2014; Newman, Howlett, \& Burton, 2014).

\section{Research on Reductive (Nutrient-Specific) Versus Evaluative (Summary) Systems}

In the case of reductive (nutrient-specific) systems, such as traffic lights, when the NFP is not available, the multi-color traffic lights result in significantly greater nutrition accuracy scores than a simple evaluative icon or control (Andrews, Burton, \& Kees, 2011) or the Facts Up Front icon (Roberto et al., 2012). Thus, the reductive, nutrient-specific icons tend to aid consumer understanding and education objectives. Interestingly, Newman, Howlett, and Burton (2016) find that reductive (nutrient specific) icons (as opposed to evaluative-summary icons) aided nutrition evaluations and purchase intentions for healthier food products when a single item was evaluated, yet these results are reversed in the case of comparing multiple food items. When the product evaluation task becomes more complex due to a larger number of products to be compared, the need and usefulness for more simplistic, evaluative icons become more beneficial for this more 
Nutrition Labeling in the United States and the Role of Consumer Processing, Message Structure, and Moderating Conditions

difficult judgment task. When the goal is to assist consumers in making healthful choices at the point-of-purchase by comparing alternative brands in a category, these evaluative icons appear most helpful.

\section{Opportunities for FOP Research}

As noted in Andrews et al. (2014, p. 14) and Newman et al. (2016, p. 764), there are many unanswered questions regarding the FOP icons and systems. For example, will FOP symbols and icons meet the needs of those with lower literacy skills? How is FOP nutrition information processed differently by the most vulnerable groups of consumers (i.e., those with diet-related conditions such as hypertension)? What might be the most effective formats, colors, size, and graphic design? How will they work in large field studies? Finally, how might nutrient-specific, objective (versus evaluative-summary) icons work in an online retail store versus in-store environment?

\section{Health and Nutrient Content Claims}

\section{Brief History of Health and Nutrient Content Claims}

The NLEA (1990) provided the FDA with the authority to require all packaged foods to bear nutrition labeling, and require that all nutrient content claims (e.g., "high fiber," "low sodium," etc.) and health claims (e.g., "a diet low in total fat may reduce the risk of cancers") be consistent with agency regulations, definitions, and standards (FDA, 1994). As indicated previously, this occurred because health and nutrition claims had become quite prevalent in the 1980s, with Kellogg's bypassing the FDA to promote a fiber-reduced cancer health claim for their All-Bran cereal. 


\section{Claim Research with NFPs and Moderating Conditions}

In addition to NFP research, there have been numerous reviews of health and nutrition claim research over the years (e.g., Caudill, 1994; GAO, 2011; Geiger, 1998; Health Canada, 2009; Hieke \& Taylor, 2012; Hasler, 2008; Lahteenmaki, 2012; Leathwood, Richardson, Sträter, Todd, \& Trijp, 2007; Levy, 2004; Nocella \& Kennedy, 2012; Pothoulaki \& Chryssochoidis, 2009; Williams, 2005). Interested readers are directed to these reviews, as we will now focus on four studies that examined interactions between the NFP and nutrition claims, as well as examples of moderating conditions in nutrition claim research.

In the first study of NFPs in the presence of health claims, Ford, Hastak, Mitra, and Ringold (1996) found that consumers were accurate in the nutritional evaluation of products from the NFP, even in the presence of contradictory health claims. (This was also found to be true with nutrient content claims in Keller et al., 1997.) As an extension of that study, Mitra, Hastak, Ford, and Ringold (1999) determined that regardless of educational levels (split by those with/without a high school degree), consumers were capable of evaluating and using the NFP even in the presence of a contradictory implied health claim. However, it has been shown that factors such as consumer numeracy can moderate how quantitative front-of package nutrition claims are processed and evaluated by consumers (Tangari, Burton, \& Davis, 2014). In addition, as pointed out by Roe, Levy, and Derby (1999), the previous designs used forced exposure when both health claims and the NFP were presented to respondents. In Roe et al. (1999), an experimental design randomly assigned respondents to a front panel for three products with either (1) no health or nutrient content claims (control), (2) only nutrient content claims, or (3) one of eight conditions that had both nutrient content claims (varying format length and wording). Importantly, they also measured information search truncation, recording if a respondent examined (1) only the package label's front panel, (2) only the package's NFP, (3) both the package's front and NFP, or (4) neither panel. The first primary finding in Roe et al. (1999) is that the presence of a health claim (e.g., folic acid-reducing neural tube defects for cereal), and to some extent a nutrient content claim, significantly increased the likelihood that respondents truncated their search to only the front panel (and not to the NFP). Second, when a health or nutrient content claim is present, respondents viewed the product as healthier and had stronger purchase intentions, both independent of their search behavior. Finally, consumers are more likely to attribute inappropriate health benefits-i.e., health halos-when a health or nutrient content claim is present (i.e., rating the product as being higher on other health attributes not mentioned in the claim). For one product (lasagna), consumers generated a magic bullet effect due to the claims (i.e., attributing inappropriate health benefits to the product).

In the study of nutrition claims, prior product perceptions and moderating conditions do matter. For example, in Andrews, Netemeyer, and Burton (1998) and Andrews, Burton, and Netemeyer (2000), a pretest from primary food shoppers first revealed that margarine was viewed as significantly less nutritious and soup was rated as significantly more nutritious 


\section{Nutrition Labeling in the United States and the Role of Consumer Processing, Message Structure, and Moderating Conditions}

from a range of products evaluated. Then, in Andrews et al. (1998), experimentally manipulated margarine ads with general ("Healthy") and specific ("Low Cholesterol") absolute nutrient content claims were overgeneralized by consumers to perceptions of healthiness and low perceived levels of other nutrients ("low in fat") when they actually were high. These misleading halos were reduced only with the use of an evaluative disclosure (i.e., characterizing the per-serving level of the margarine to be "high" as evaluated by the FDA) that was effective regardless of nutrition knowledge levels. In contrast, as found in Andrews et al.'s (2000) study of general ("Healthier") and specific ("1/3 less sodium") relative nutrient content claims for soup, although misleading halos to absolute sodium levels occurred, the effects of disclosures in reducing such halos were found to be dependent on objective nutrition knowledge and claim type. Thus, in the case of products such as soup that are perceived as more "nutritious" (yet with high levels of negative nutrient), prior nutrition knowledge may be needed for disclosures to work. This also is the case for specific types of nutritional knowledge (Andrews, Netemeyer, \& Burton, 2009; Wansink, 2005). For example, Andrews et al. (2009) found a significant curvilinear (quadratic) relationship for the effects of caloric knowledge, obesity consequences knowledge, and motivation to search for nutrition information on purchase intentions for an advertised, high-calorie snack bar with lower fat and lower calorie claims. In this study, intent to buy the high-calorie snack bar was significantly reduced, yet only at the highest levels of caloric knowledge, obesity consequences knowledge, and nutrition search motivation. Also, cognitive process checks and regression findings indicated that exposure to the relative nutrition ad claims led to health halo effects. 


\section{Some Claims Not Regulated by the FDA}

While the FDA regulates many nutrient content and health claims, others are not regulated or are regulated by other agencies (e.g., organic claims and nutrition information for meat and poultry products by the USDA). Perhaps the greatest need for research on nutrition claims today involves these sets of claims not regulated by the FDA (e.g., "organic," "natural," "gluten-free," "GMO-free") and how they may interact with the revised (new) NFPs and FOPs, as well as under different moderating conditions examined in previous claim research. A recent example of a claim that has not been defined or regulated by the FDA is the use of "natural" or "all-natural" claims. Other alternative food claims include features that relate to ethicality (e.g., fair trade, cage-free, GMO-free), health or diet (e.g., gluten or lactose-free), safety (e.g., traceability), religion (e.g., kosher, halal), and economics (e.g., locally grown, community-supported), and many of these claims are still emerging (USDA, 2014). Two challenges regarding the effectiveness of such claims include definition and understanding. While a definition for "certified organic" has been created and managed by the USDA, unregulated claims ("natural") often lack a common definition and consistent practice by food providers. Consumers' understanding of these claims, then, is limited due to their complexity and varied interpretations (Verbeke \& Ward, 2006). A "gluten-free" claim may appear straightforward, yet many manufacturers have different practices for production (e.g., segregation of containers with gluten from those without). The negative implications for those suffering from celiac disease, then, are tremendous. Even for the more common claims of "organic" and "GMOfree," consumers continue to have a poor understanding of these terms and do not consider them relevant in their product decisions (Stanton \& Cook, 2015). Recently, legislation was signed in law that requires food packages to display an electronic (QR) code, text label, or some sort of symbol signifying whether or not they contain GMOs (Haddon, 2016). The exact details will need to be worked out by the USDA, which will have up to two years to write the rules.

There has been a growing demand for natural food as evidenced by the fact products labeled as natural have grown by $24 \%$ over recent years (Nielsen, 2015). Given the increasing and high level of consumer demand for products labeled as natural, many of these products are associated with significant price premiums (USDA, 2014). Yet, the FDA has refused to offer regulations defining exactly what "natural" means on a package label, and thus many consumers rely on their own assumptions about what this labeling infers when evaluating products and making choices decisions. While there is minimal academic research, some recent findings contend that these inferences drawn (e.g., including the likelihood that the product is minimally processed, free of GMOs, or likely to be an organic offering) may mislead consumers and have a direct or indirect influence on choices (Berry, Burton, \& Howlett, FoRTHCOMING). Since natural claims are unregulated by the FDA, a food product with a natural claim prominently displayed on the package 
Nutrition Labeling in the United States and the Role of Consumer Processing, Message Structure, and Moderating Conditions

label may, in fact, not be organic, minimally processed, and free of GMOs and artificial ingredients. Thus, natural claims are potentially misleading if consumers' product evaluations and choice decisions are influenced by false inferences.

Because the FDA has not defined or regulated use of the "natural" claim, there has been a substantial amount of litigation regarding the potentially misleading use of "natural" claims. Petty (2015) reports that from 2011 to 2013, there were some 200 class action lawsuits filed related to the possibility of deceptive use of "natural" claims, and the increase was dramatic compared to the prior eight years. Given the uncertainty in the marketplace, there have been multiple consumer petitions to the FDA, and the agency recently has formally asked consumer and manufacturers to provide information and comments on the use of the term "natural" on food products (Federal Register, 2015).

In contrast to the unregulated natural claim by the FDA, the USDA organic label has been included on food labels since 2002 (USDA, 2016C) and is managed by the USDA's Agricultural Marketing Service (AMS). Based on USDA criteria, organic foods are grown and processed according to requirements, including the restrictions on the use of additives and artificial ingredients, pesticides, soil quality, and animal raising and processing practices (USDA, 2012). For composite packaged food products, all ingredients and processing aids must be certified organic for an organic claim to be used (USDA, 2012). Given the definition and labeling requirements in use for more than a decade, most consumers have a broad general understanding of what the organic label means for a product (e.g., Consumer Reports National Research Center, 2014). In general, the use of the term "organic" in addition to the inclusion of the USDA-certified organic seal mirror other alternative food market level practices. The direct marketing alternative food source model (which includes the organic industry) is one of the largest and fastest growing in agribusiness (Venn et al., 2006). As a result, research in this context or for other unregulated claims could explore how and to what extent consumers use this information to inform their product decisions. Since alternative foods, such as organic foods, are believed to be inherently more healthful (Little et al., 2009; SAGE, 2003), vulnerability to misinterpretation and overgeneralization warrants further examination.

In general, however, and in contrast to research on natural claims, the organic label, and the breadth of its influence, has been the subject of considerable research across various academic disciplines. However, one topic for future research could relate to direct comparisons between natural and organic claims on food packaging. It seems likely that some marketers may be sidestepping the somewhat precise and demanding process for organic status by using a natural claim instead. Some surveys suggest that "natural" claims are more persuasive than "organic" claims, with "natural" claims rated as important or very important by consumers, with 35\% rating organic claims as important or very important (Negowetti, 2013; Petty, 2015). Due to the specific definition and requirements for "organic," but not for "natural," claims research that addressed comparisons of manipulations of organic, natural, and no claim control conditions on 


\section{Conclusions and Future Research on Nutrition Labeling Issues in the United States}

Clearly, due to the many recent and forthcoming changes in the nutrition labeling environment, there are many unanswered questions on nutrition label effects that offer many future research opportunities. These include understanding the effectiveness of the imminent changes in the marketplace, including the following:

1) The specific changes to the revised NFP (e.g., added sugars, more prominent display of calories) and how it will impact the broad population and consumer segments with more specific health risks;

2) The long-term effects of beef and poultry labeling and how changes in the communication of the information may impact its effects on evaluations and consumption, as well as considering what factors moderate effects;

3) Potential effects of the labeling of seafood, which often has a more favorable nutrition profile but less usage in the U.S. than many meat alternatives;

4) Effects of calorie labeling for restaurant chains on consumer restaurant evaluations and meal choices;

5) The impact of the forthcoming labeling on other retail institutions serving foods for immediate consumption, including movie theaters, bakeries, grocery stores, ice cream shops, and convenience stores;

6) Effects of alternative front-of-package formats, nutrient content, and health claims and how each may interact with new NFP formats; and

7) Additional research on currently unregulated claims receiving substantial attention in the marketplace and scrutiny by U.S. federal agencies (e.g., natural claims, GMO-free, gluten-free).

Each of the above topics offers the potential for meaningful changes that improve consumers' long-term choices and can positively affect health and consumer well-being. Yet, research cited here has demonstrated that information provision does not always guarantee favorable changes in consumer choices and, at times, can lead to unintended consequences that were not considered when developing policies. Conversely, habitual decisions and behavior change may occur without much thought. We also encourage researchers to examine how different labeling information (e.g., claims, front-of-pack symbols, nutrition facts) might interact under differing conditions, such as one's motivation, ability, and/or opportunity to process information. This all points to the need for broader research into the conditions important in influencing a wider range of 
Nutrition Labeling in the United States and the Role of Consumer Processing, Message Structure, and Moderating Conditions

consumer outcomes (e.g., awareness to comprehension to behavior) that may occur over time with the provision of nutrition information.

\section{Further Reading}

Derby, B. M., \& Levy, A. S. (2001). Do food labels work? Gauging the effectiveness of food labels pre- and post-NLEA. In P. N. Bloom \& G. T. Gundlach (Eds.), Handbook of marketing and society (pp. 372-398). Thousand Oaks, CA: SAGE.

Drichoutis, A. C., Lazaridis, P., \& Nayga, R. M., Jr. (2006). Consumers' use of nutritional labels: A review of research studies and issues. Academy of Marketing Science Review, 9, 93-108.

FDA. (1994). Guidance for industry: A food labeling guide. College Park, MD: U.S. Food and Drug Administration.

FDA. (2016a, May 20). FDA modernizes nutrition facts label for packaged foods. Retrieved from http://www.fda.gov/NewsEvents/Newsroom/PressAnnouncements/ ucm502182.htm.

Geiger, C. J. (1998). Health claims: History, current regulatory status, and consumer research. Journal of the American Dietetic Association, 98(11), 1312-1322.

Hieke, S., \& Taylor, C. R. (2012). A critical review of the literature on nutritional labeling. The Journal of Consumer Affairs, 46, 120-156.

Institute of Medicine (IOM). (2011, October 18). Examination of front-of-package nutrition rating systems and symbols. Retrieved from http://www.iom.edu/Activities/ Nutrition/NutritionSymbols.aspx.

Keller, S., Landry, M., Olson, J., Velliquette, A., Burton, S., \& Andrews, J. C. (1997). The effects of nutrition package claims, nutrition facts panels, and motivation to process nutrition information on consumer product evaluations. Journal of Public Policy \& Marketing, 16, 256-269.

Long, M. W., Tobias, D. K., Cradock, A. L., Batchelder, H., \& Gortmaker, S. L. (2015). Systematic review and meta-analysis of the impact of restaurant menu calorie labeling. American Journal of Public Health, 105(5), 11-24.

Wansink, B., \& Chandon, P. (2014). Slim by design: Redirecting the accidental drivers of mindless overeating. Journal of Consumer Psychology, 24(3), 413-431. 


\section{References}

American Heart Association (AHA). (2014). Heart-Check Food Certification Program. Retrieved from http://www.heart.org/HEARTORG/GettingHealthy/Nutrition-Center/ HeartSmartShopping/Heart-Check-Food-CertificationProgram:UCM_300133_Article.jsp.

Andrews, J. C. (2011). Warnings and disclosures. In B. Fischoff, N. Brewer, \& J. Downs (Eds.), Communicating risk and benefits: An evidence-based user's guide (pp. 149-161). Silver Spring, MD: U.S. Food \& Drug Administration.

Andrews, J. C., Burton, S., \& Kees, J. (2011). Is simpler always better? Consumer evaluations of front-of-package nutrition symbols. Journal of Public Policy \& Marketing, 30, 175-190.

Andrews, J. C., Burton S., \& Netemeyer, R. G. (2000). Are some comparative nutrition claims misleading? The role of nutrition knowledge, ad claim type, and disclosure conditions. Journal of Advertising, 29, 29-42.

Andrews, J. C., Lin, C-T., Levy, A. S., \& Lo, S. (2014). Consumer research needs from the Food and Drug Administration on front-of-package nutritional labeling. Journal of Public Policy \& Marketing, 33(1), 10-16.

Andrews, J. C., Netemeyer, R. G., \& Burton, S. (1998). Consumer generalization of nutrient content claims in advertising. Journal of Marketing, 62, 62-75.

Andrews, J. C., Netemeyer, R. G., \& Burton, S. (2009). The nutrition elite: Do only the highest levels of caloric knowledge, obesity knowledge, and motivation matter in processing nutrition ad claims and disclosures. Journal of Public Policy \& Marketing, 28, 41-55.

Asam, E. H., \& Bucklin, L. P. (1973). Nutritional labeling for canned goods: A study of consumer response. Journal of Marketing, 37, 32-37.

Barone, M. J., Rose, R. L., Manning, K. C., \& Miniard, P. W. (1996). Another look at the impact of reference information on consumer impressions of nutrition information. Journal of Public Policy \& Marketing, 15(1), 55-62.

Bates, K., Burton, S., Howlett, E., \& Huggins K. (2009). The roles of gender and motivation as moderators of the effects of calorie and nutrient information provision on away-from-home-foods. Journal of Consumer Affairs, 43, 249-273.

Baumeister, R. F., Bratslavsky, E., Finkenauer, C., \& Vohs, K. D. (2001). Bad is stronger than good. Review of General Psychology, 5, 323-370. 
Nutrition Labeling in the United States and the Role of Consumer Processing, Message Structure, and Moderating Conditions

Berry, C., Burton, S., \& Howlett, E. (forthcoming). It's only natural: The mediating impact of consumers' inferential beliefs on the relationships between product claims, perceived product healthfulness, and purchase intentions. Journal of the Academy of Marketing Science.

Bi, X., House, L., \& Gao, Z. (2016). Impacts of nutrition information on choices of fresh seafood among parents. Marine Resource Economics, 31(3), 182.

Block, L. G., \& Peracchio, L. A. (2006). The calcium quandary: How to use nutrition labels. Journal of Public Policy \& Marketing, 25(20), 188-196.

Bollinger, B., Leslie, P., \& Sorensen A. (2011). Calorie posting in chain restaurants. American Economic Journal: Economic Policy, 3(1), 91-128.

Brucks, M., Mitchell, A. A., \& Staelin R. (1984). The effect of nutritional information disclosure in advertising: An information processing approach. Journal of Public Policy \& Marketing, 3(1), 1-25.

Burke, S. J., Milberg, S. J., \& Moe W. W. (1997). Displaying common but previously neglected health claims on product labels: Understanding competitive advantages, deception, and education. Journal of Public Policy \& Marketing, 16, 242-255.

Burros, M. (1986, February 19). Health claims on food put F.D.A. in a corner. The New York Times. Retrieved from http://www.nytimes.com/1986/02/19/garden/healthclaims-on-food-put-fda-in-a-corner.html?pagewanted=all.

Burton, S., \& Andrews, J. C. (1996). Age, product nutrition, and label format effects on consumer perceptions and product evaluations. The Journal of Consumer Affairs, 30(1), 68-89.

Burton, S., Andrews, J. C., \& Netemeyer, R. G. (2000). Nutrition ad claims and disclosures: Interaction and mediation effects for consumer evaluations of the brand and the ad. Marketing Letters, 11(3), 235-247.

Burton, S., Biswas, A., \& Netemeyer, R. (1994). Effects of alternative nutrition label formats and nutrition reference information on consumer perceptions, comprehension, and product evaluations. Journal of Public Policy \& Marketing, 13(1), 36-47.

Burton, S., Cook, L. A., Howlett, E., \& Newman C. (2015). Broken halos and shattered horns: Overcoming the biasing effects of prior expectations through objective information disclosure. Journal of the Academy of Marketing Science, 43(2), 240-256.

Burton, S., Creyer, E. H., Kees, J., \& Huggins, K. (2006). Attacking the obesity epidemic: The potential health benefits of providing nutrition information in restaurants. American Journal of Public Health, 96(9), 1669-1675. 
Nutrition Labeling in the United States and the Role of Consumer Processing, Message Structure, and Moderating Conditions

Burton, S., Garretson, J. A., \& Velliquette, A.M. (1999). Implications of accurate usage of nutrition facts panel and information for food product evaluations and purchase intentions. Journal of the Academy of Marketing Science, 27(4), 470-481.

Burton, S., Howlett, E., \& Tangari, A. H. (2009). Food for thought: How will the nutrition labeling of quick service restaurant menu items influence consumers' product evaluations, purchase intentions, and choices? Journal of Retailing, 85(3), 258-273.

Burton, S., \& Kees, J. (2012). Flies in the ointment? Addressing potential impediments to population-based health benefits of restaurant menu labeling initiatives. Journal of Public Policy \& Marketing, 31(2), 232-239.

Burton, S., Tangari, A., Howlett, E., \& Turri, A. (2014). How the perceived healthfulness of restaurant menu items influences sodium and calorie misperceptions: Implications for nutrition disclosures in chain restaurants. Journal of Consumer Affairs, 48, 62-95.

Caudill, E. M. (1994). Nutritional information research: A review of the issues. Advances in Consumer Research, 21, 213-217.

Chandon, P., \& Wansink, B. (2007). The biasing health halos of fast-food restaurant health claims: Lower calorie estimates and higher side-dish consumption intentions. Journal of Consumer Research, 34, 301-314.

Chiuve, S. E., Fung, T. T., Rimm, E. B., Hu, F. B., McCullough, M. L., Wang, M., et al. (2012). Alternative dietary indices both strongly predict risk of chronic disease. The Journal of Nutrition, 142(6), 1009-1018.

Consumer Reports National Research Center. (2014). Organic food labels survey: 2014 nationally-representative phone survey. Retrieved from http://www.greenerchoices.org/ pdf/CR2014OrganicFoodLabelsSurvey.pdf.

Cook, L. A., Burton, S., \& Howlett, E. (2011). Health risk factors and their effect on consumers' use of nutrition facts panels. Journal of Consumer Affairs, 45, 516-527.

Cook, L. A., Burton, S., \& Howlett E. (2013). Leaner choices? The potential influence of the inclusion of nutrition facts panels on consumer evaluations and choices of ground beef products. Journal of Public Policy \& Marketing, 32, 97-115.

Cook, T. D., \& Campbell, D. T. (1979). Quasi-experimentation: Design \& analysis issues for field settings. Boston: Houghton Mifflin.

Center for Science in the Public Interest. (2006, November 30). FDA urged to create new "healthy food" labeling system. Retrieved from http://www.cspinet.org/new/

\section{1.html.}

Daniel, C. R., Cross, A. J., Koebnick, C., \& Sinha, R. (2011). Trends in meat consumption in the United States. Public Health Nutrition, 14, 575-583. 
Nutrition Labeling in the United States and the Role of Consumer Processing, Message Structure, and Moderating Conditions

Derby, B. M., \& Levy, A. S. (2001). Do food labels work? Gauging the effectiveness of food labels pre- and post-NLEA. In P. N. Bloom \& G. T. Gundlach (Eds.), Handbook of marketing and society (pp. 372-398). Thousand Oaks, CA: SAGE.

Derby, B. M., \& Levy, A. S. (2005). Effects of strength of science disclaimers on the communication impacts of health claims. Working Paper No. 1. College Park, MD: U.S. Food and Drug Administration. Retrieved from http://www.fda.gov/OHRMS/dockets/ dockets/03N0496/03N-0496-rpt0001.pdf.

Downs, J. S., Wisdom, J., Wansink, B., \& Loewenstein, G. (2013). Supplementing menu labeling with calorie recommendations to test for facilitation effects. American Journal of Public Health, 103(9), 1604-1609.

Dumanovsky, T., Huang, C. Y., Nonas, C. A., Matte, T. D., Bassett, M. T., \& Silver, L. D. (2011). Changes in energy content of lunchtime purchases from fast food restaurants after introduction of calorie labelling: Cross sectional customer surveys. BMJ: British Medical Journal, 343, d4464.

Elbel, B., Gyamfi, J., \& Kersh, R. (2011). Child and adolescent fast food choice and the influence of calorie labeling: A natural experiment. International Journal of Obesity, 35(4), 493-500.

Elbel, B., Kersh, R., Brescoll, V. L., \& Dixon, L. B. (2009). Calorie labeling and food choices: A first look at the effects on low-income people in New York City. Health Affairs, 28(6), 1110-1121.

Ellison, B., Lusk, J. L., \& Davis, D. (2013). The effect of calorie labels on caloric intake and restaurant revenue: Evidence from two full-service restaurants. Journal of Agricultural and Applied Economics, 46(2), 173-191.

FDA. (1938). Federal Food, Drug, and Cosmetic Act, Public Law No. 75-717, 52 Stat. 1040 (codified at 21 USC).

FDA. (1994). Guidance for industry: A food labeling guide. College Park, MD: U.S. Food and Drug Administration.

FDA. (2003a, July 10). Consumer health information for better nutrition initiative, task force final report. Washington, DC: U.S. Food and Drug Administration. Retrieved from http://www.fda.gov/ohrms/dockets/dockets/06p0243/06p-0243-cp00001-005Attachment-02.pdf.

FDA. (2003b, July 10). Guidance for Industry and FDA: Interim Evidence-based Ranking System for Scientific Data. Retrieved from http://www.fda.gov/Food/ GuidanceRegulation/GuidanceDocumentsRegulatoryInformation/ LabelingNutrition/ucm073332.htm. 
Nutrition Labeling in the United States and the Role of Consumer Processing, Message Structure, and Moderating Conditions

FDA. (2003c). Food labeling: Health claims; dietary guidance, proposed rule. Federal Register, 68(227), 66040-66048.

FDA. (2016a, May 20). FDA modernizes nutrition facts label for packaged foods. Retrieved from http://www.fda.gov/NewsEvents/Newsroom/PressAnnouncements/ ucm502182.htm.

FDA. (2016b). Eye tracking and experimental tests of NFP formats as part of the Federal Register notice of reopening of the comment period. Retrieved from http:// www.regulations.gov/\# !documentDetail;D=FDA-2012-N-1210-0552; http:// www.regulations.gov/\# !documentDetail;D=FDA-2012-N-1210-0553; and testing of added sugars and footnote provisions of the revised NFPs as part of the Supplemental Notice of Proposed Rulemaking http://www.regulations.gov/\#! documentDetail;D=FDA-2012-N-1210-0539, http://www.regulations.gov/\# ! documentDetail;D=FDA-2012-N-1210-0540.

Federal Register. (2010). Nutrition labeling of single-ingredient products and ground or chopped meat and poultry products: Final rule, 75(249), 82148-82167.

Federal Register. (2015). Use of the term "natural" in the labeling of human food products: Request for information and comments, 80, 69905-69909.

Finkelstein, E. A., Strombotne, K. L., Chan, N. L., \& Krieger, J. (2011). Mandatory MENU LABELING IN ONE FAST-FOOD CHAIN in King County, Washington. American Journal of Preventive Medicine, 40(2), 122-127.

Ford, G. T., Hastak, M., Mitra, A., \& Ringold, D. J. (1996). Can consumers interpret nutrition information in the presence of a health claim? A laboratory investigation. Journal of Public Policy \& Marketing, 15(1), 16-27.

Freiden, J. B. (1981). The effect of nutrition information on brand rating: Test for a nonuse benefit. The Journal of Consumer Affairs, 15(1), 106-114.

GAO. (2011, January). Food labeling: FDA needs to reassess its approach to protecting consumers from false or misleading claims. Report to Congressional Committee (pp. 155). Washington, DC: United States Government Accountability Office.

Garretson, J. A., \& Burton, S. (2000). Effects of nutrition facts panel values, nutrition claims, and health claims on consumer attitudes, perceptions of disease-related risks, and trust. Journal of Public Policy \& Marketing, 19(2), 213-227.

Geiger, C. J. (1998). Health claims: History, current regulatory status, and consumer research. Journal of the American Dietetic Association, 98(11), 1312-1322.

Gonzalez-Vallejo, C., \& Lavins, B. D. (2015). Evaluation of breakfast cereals with the current nutrition facts panel (NFP) and the Food and Drug Administration's NFP proposal. Public Health Nutrition, 19(6), 1047-1058. 
Nutrition Labeling in the United States and the Role of Consumer Processing, Message Structure, and Moderating Conditions

Gonzalez-Vallejo, C., Lavins, B. D., \& Carter, K. A. (2016). Analysis of nutrition judgements using the nutrition facts panel. Appetite, 105, 71-84.

Graham, D. J., \& Roberto, C. A. (2016). Evaluating the impact of U.S. Food and Drug Administration-proposed nutrition facts label changes on young adults' visual attention and purchase intentions. Health Education \& Behavior, 43(4), 389-398.

Grocery Manufacturers of America (GMA). (2011, January 24). Food and beverage industry launches nutrition keys front-of-pack nutrition labeling initiative to inform consumers and combat obesity. Press release. Retrieved from http:// www.gmaonline.org/news-events/newsroom/food-and-beverage-industrylaunches-nutrition-keys-front-of-pack-nutrition-.

Grocery Manufacturers of America (GMA) and Food Marketing Institute (FMI). (2011, January 24). Nutrition keys: Front-of-package nutrition labeling initiative. Facts Sheet, 12 .

Haddon, H. (2016, August 4). Nutrition labels you'll need a smartphone to read. The Wall Street Journal, D1-D4.

Hasler, C.M. (2008). Health claims in the United States: An aid to the public or a source of confusion? The Journal of Nutrition, 138(June), 1216S-1220S.

Health Canada. (2009). Consumer understanding of health claims-qualitative research. HC POR 08-16. Toronto: Health Canada.

Hieke, S., \& Taylor, C. R. (2012). A critical review of the literature on nutritional labeling. The Journal of Consumer Affairs, 46, 120-156.

Howlett, E. A., Burton, S., Bates, K., \& Huggins, K. (2009). Coming to a restaurant near you? Potential consumer responses to nutrition information disclosure on menus. Journal of Consumer Research, 36, 494-503.

Howlett, E. A., Burton, S., \& Kozup, J. (2008). How modification of the nutrition facts panel influences consumers at risk for heart disease: The case of trans fat. Journal of Public Policy \& Marketing, 21(1), 83-97.

Howlett, E. A., Burton, S., Tangari, A., \& Bui, M. (2012). Hold the salt! Effects of sodium information provision, sodium content, and hypertension on perceived cardiovascular disease risk and purchase intentions. Journal of Public Policy \& Marketing, 31, 4-18.

Institute of Medicine (IOM). (2011, October 18). Examination of front-of-package nutrition rating systems and symbols. Retrieved from http://www.iom.edu/Activities/ Nutrition/NutritionSymbols.aspx.

Jacoby, J., Chestnut, R. W., \& Silberman, W. (1977). Consumer use and comprehension of nutrition information. Journal of Consumer Research, 4, 119-128. 
Kees, J., Burton, S., \& Andrews, J. C. (2015). Government efforts to aid consumers' wellbeing: Understanding federal health warnings and disclosures. In M. Norton, D. Rucker \& C. Lamberton (Eds.), The Cambridge handbook of consumer psychology (pp. 530-562). New York: Cambridge University Press.

Keller, S., Landry, M., Olson, J., Velliquette, A., Burton, S., \& Andrews, J. C. (1997). The effects of nutrition package claims, nutrition facts panels, and motivation to process nutrition information on consumer product evaluations. Journal of Public Policy \& Marketing, 16, 256-269.

Kemp, E., Burton, S., Creyer, E. H., \& Suter, T. A. (2007). When do nutrient content and nutrient content claims matter? Assessing consumer tradeoffs between carbohydrates and fat. The Journal of Consumer Affairs, 41(1), 47-73.

Kozup, J. C., Creyer, E. H., \& Burton, S. (2003). Making healthful food choices: The influence of health claims and nutrition information on consumers' evaluations of packaged food products and restaurant menu items. Journal of Marketing, 67, 19-34.

Krieger, J. W., Chan, N. L., Saelens, B. E., Ta, M. L., Solet, D., \& Fleming, D. W. (2013). Menu labeling regulations and calories purchased at chain restaurants. American Journal of Preventive Medicine, 44(6), 595-604.

Lahteenmaki, L. (2012). Claiming health in food products. Food Quality and Preference, 27(2), 196-201.

Laquatra, I., Sollid, K., Edge, M. S., Pelzel, J., \& Turner, J. (2015). Including "added sugars" on the nutrition facts panel: How consumers perceive the proposed change. Journal of the Academy of Nutrition and Dietetics, 115(11), 1758-1763.

Leathwood, P. D., Richardson, D. P., Sträter, P., Todd, P. M., \& van Trijp, H. C. (2007). Consumer understanding of nutrition and health claims: Sources of evidence. British Journal of Nutrition, 98, 474-484.

Lenahan, R. J., Thomas, J. A., Taylor, D. A., Call, D. L., \& Padberg, D. I. (1973). Consumer reaction to nutritional labels on food products. The Journal of Consumer Affairs, 7(1), 112.

Levy, A. S. (2004). Consumer research about health claims on food/dietary supplement product labeling. College Park, MD: U.S. Food and Drug Administration.

Levy, A. S., Fein, S. B., \& Schucker, R. E. (1996). Performance characteristics of seven nutrition label formats. Journal of Public Policy \& Marketing, 15, 1-15.

Levy, A. S., Mathews, O., Stephenson, M., Tenner, J. E., \& Schucker, R. E. (1985). The impact of a nutrition information program on food purchases. Journal of Public Policy \& Marketing, 4(1), 1-13. 
Nutrition Labeling in the United States and the Role of Consumer Processing, Message Structure, and Moderating Conditions

Li, F., Miniard, P. W., \& Barone, M. J. (2000). The facilitating influence of consumer knowledge on the effectiveness of daily value reference information. Journal of the Academy of Marketing Science, 28(3), 425-436.

Little, J., Ilbery, B. \& Watts, D. (2009). Gender, consumption and the relocalisation of food: A research agenda. Sociologia Ruralis, 49(3), 201-217.

Long, M. W., Tobias, D. K., Cradock, A. L., Batchelder, H., \& Gortmaker, S. L. (2015). Systematic review and meta-analysis of the impact of restaurant menu calorie labeling. American Journal of Public Health, 105(5), 11-24.

Malik, V. S., Willett, W. C., \& Hu, F. B. (2016). The revised nutrition facts label: A step forward and more room for improvement. Journal of the American Medical Association, 316(1), 583-584.

Mazis, M. B., \& Raymond, M. A. (1997). Consumer perceptions of health claims in advertisements and on food labels. The Journal of Consumer Affairs, 31(1), 10-26.

Mitra, A., Hastak, M., Ford, G. T., \& Ringold, D. J. (1999). Can the educationally disadvantaged interpret the FDA-mandated nutrition facts panel in the presence of an implied health claim? Journal of Public Policy \& Marketing, 18, 106-117.

Moorman, C. (1990). The effects of stimulus and consumer characteristics on the utilization of nutrition information. Journal of Consumer Research, 17, 362-374.

Moorman, C. (1996). A quasi experiment to assess the consumer and informational determinants of nutrition information processing activities: The case of the Nutrition Labeling and Education Act. Journal of Public Policy \& Marketing, 15(1), 28-44.

Muller, T. E. (1985). Structural information facts which stimulate the use of nutrition information: A field experiment. Journal of Marketing Research, 22, 143-157.

Negowetti, N. E. (2013). A national "natural" standard for food labeling. Maine Law Review, 65, 581.

Nelson, D., Graham, D., \& Harnack, L. (2014). An objective measure of nutrition facts panel usage and nutrient quality of food choice. Journal of Nutrition Education and Behavior, 46(6), 589-594.

Newman, C. L., Howlett, E., \& Burton, S. (2014). Shopper response to front-of-package nutrition labeling programs: Potential consumer and retail store benefits. Journal of Retailing, 90(1), 13-26.

Newman, C. L., Howlett, E., \& Burton, S. (2016). The effects of objective and evaluative front-of-package cues on food evaluation and choice: The moderating influence of comparative and non-comparative processing contexts. Journal of Consumer Research, $42,749-766$. 
Nutrition Labeling in the United States and the Role of Consumer Processing, Message Structure, and Moderating Conditions

Nielsen. (2015). We are what we eat: Healthy eating trends around the world. Retrieved from http://www.nielsen.com/content/dam/nielsenglobal/eu/nielseninsights/pdfs/ Nielsen\%20Global\%20Health\%20and\%20Wellness\%20Report\%20\%20January\%202015.pdf.

NHANES. (2007-2008). National Health and Examination Study, Atlanta, GA: Centers for Disease Control and Prevention. Retrieved from http://wwwn.cdc.gov/nchs/nhanes/ search/nhanes07_08.aspx.

NLEA. (1990). Nutrition Labeling Education Act, Public Law No. 101-535, 104 Stat. 2353 (codified in part at 21 U.S.C. 343).

Nocella, G., \& Kennedy, O. (2012). Food health claims-what consumers understand. Food Policy, 37, 571-580.

NuVal. (2014). .ONQI: The science behind the scores. Retrieved from http:// www.nuval.com/science.

Parker, J. R., \& Lehmann, D. L. (2014). How and when grouping low-calorie options reduces the benefits of providing dish-specific calorie information. Journal of Consumer Research, 41(1), 213-235.

Petty, R. (2015). "Natural” claims in food advertising: Policy implications of filling the regulatory void with consumer class action lawsuits. Journal of Public Policy \& Marketing, $34,131-141$.

Petty, R. E., \& Cacioppo, J. T. (1986). Communication and persuasion: Central and peripheral routes to attitude change. New York: Springer.

Porter, D. V., \& Earl, R. O. (Eds.). (1990). Nutrition labeling: Issues and directions for the 1990s, Washington, DC: National Academy Press.

Pothoulaki, M., \& Chryssochoidis, G. (2009). Health claims: Consumers' matters. Journal of Functional Foods, 1, 222-228.

Raghunathan, R., Naylor, R. W., \& Hoyer, W. D. (2006). The unhealthy = tasty intuition and its effects on taste inferences, enjoyment, and choice of food products. Journal of Marketing, 70(4), 170-184.

Roberto, C. A., Bragg, M. A., Schwartz, M. B., Seamans, M. J., Musicus, A., Novak, N., et al. (2012). Facts up front versus traffic light food labels: A randomized control trial. American Journal of Preventive Medicine, 43(2), 134-141.

Roberto, C. A., Schwartz, M. B., \& Brownell, K. D. (2009). Rationale and evidence for menu-labeling legislation. American Journal of Preventive Medicine, 37(6), 546-551. 
Nutrition Labeling in the United States and the Role of Consumer Processing, Message Structure, and Moderating Conditions

Roe, B., Levy, A. S., \& Derby, B. M. (1999). The impact of health claims on consumer search and product evaluation outcomes: Results from FDA experimental data. Journal of Public Policy \& Marketing, 18, 89-105.

Russo, J. E., Staelin, R., Nolan, C. A., Russell, G. J., \& Metcalf, B. L. (1986). Nutrition information in the supermarket. Journal of Consumer Research, 13, 48-70.

Sage, C. (2003). Social Embeddedness and Relations of Regard: Alternative 'Good Food' Networks in South-West Ireland. Journal of Rural Studies, 19(1), 47-60.

Scammon, D. L. (1977). "Information overload" and consumers. Journal of Consumer Research, 4, 148-155.

Sebolt, A. (2008). Standardized nutrition: Food business groups seek uniform symbols on packaging. Food Business News, June 10. Retrieved from http:// www.foodbusinessnews.net/feature_stories_print.asp?ArticleID=94121.

Stanton, J. V., \& Cook, L. A. (2015). Label heuristics or detailed processing? Choice factors for alternative foods. Proceedings of the 40th Annual Macromarketing Conference. Chicago, pp. 394-397.

Szykman, L. R., Bloom, P. N., \& Levy, A. S. (1997). A proposed model of the use of package claims and nutrition labels. Journal of Public Policy \& Marketing, 16, 228-241.

Tandon, P. S., Zhou, C., Chan, N. L., Lozano, P., Couch, S. C., Glanz, K., et al. (2011). The impact of menu labeling on fast-food purchases for children and parents. American Journal of Preventive Medicine, 41(4), 434-438.

Tangari, A., Burton, S., \& Davis, C. (2014). Do they have your number? Understanding the moderating role of format effects and consumer numeracy for quantitative front-ofpackage nutrition claims. Journal of Consumer Affairs, 48(3), 620-633.

Tversky, A., \& Kahneman, D. (1981). The framing of decisions and the psychology of choice. Science, $211,453-458$.

USDA. (2012). Labeling organic products. Washington, DC: Agricultural Marketing Service. Retrieved from https://www.ams.usda.gov/sites/default/files/media/ Labeling\%200rganic\%20Products\%20Fact\%20Sheet.pdf.

USDA. (2014, May 27). Food labeling and label approval. Washington, DC: U.S. Department of Agriculture. Retrieved from http://www.fsis.usda.gov/wps/portal/fsis/ topics/regulatory-compliance/labeling.

USDA. (2016a, July 8). U.S. beef and cattle industry: Background statistics and information, Washington, DC: U.S. Department of Agriculture. Retrieved from http:// www.ers.usda.gov/topics/animal-products/cattle-beef/statistics-information.aspx. 
Nutrition Labeling in the United States and the Role of Consumer Processing, Message Structure, and Moderating Conditions

USDA. (2016b, July 8). U.S. poultry production and value 2015 summary. Washington, DC: U.S. Department of Agriculture. Retrieved from http://usda.mannlib.cornell.edu/usda/ current/PoulProdVa/PoulProdVa-04-28-2016.pdf.

USDA. (2016c, July 30). Organic agriculture. Washington, DC: U.S. Department of Agriculture. Retrieved from http://www.usda.gov/wps/portal/usda/usdahome? contentidonly=true $\&$ contentid $=$ organic-agriculture.html.

Venkatesan, M., Lancaster, W., \& Kendall, K. W. (1986). An empirical study of alternative formats for nutritional information disclosure in advertising. Journal of Public Policy \& Marketing, 5(1), 29-43.

Venn, L., Kneafsey, L., Holloway, R., Cox, R., Dowler, E., \& Tuomainen, H. (2006). Researching European "alternative" food networks: some methodological considerations, Area, 38(3), 248-258.

Verbeke, W., \& Ward, R. W. (2006). Consumer interest in information cues denoting quality, traceability and origin: An application of ordered probit models to beef labels. Food Quality and Preference, 17(6), 453-467.

Viswanathan, M. (1994). The influence of summary information in the usage of nutrition information. Journal of Public Policy \& Marketing, 13(1), 48-60.

Viswanathan, M., \& Hastak, M. (2002). The role of summary information in facilitating consumers' comprehension of nutrition information. Journal of Public Policy \& Marketing, 21(2), 305-318.

Viswanathan, M., Hastak, M., \& Gau, R. (2009). Understanding and facilitating the usage of nutritional labels by low-literate consumers. Journal of Public Policy \& Marketing, 28(2), 135-145.

Wansink, B. (2003). How do front and back package labels influence beliefs about health claims? The Journal of Consumer Affairs, 37, 305-316.

Wansink, B. (2005). Marketing nutrition: Soy, functional foods, biotechnology, and obesity. Urbana: University of Illinois Press.

Wansink, B., \& Chandon, P. (2006). Can "low-fat" nutrition labels lead to obesity? Journal of Marketing Research, 43, 605-617.

Wansink, B., \& Chandon, P. (2014). Slim by design: Redirecting the accidental drivers of mindless overeating. Journal of Consumer Psychology, 24(3), 413-431.

White House Conference on Food, Nutrition, and Health (1970). Final Report. Washington, DC: U.S. Government Printing Office. Retrieved from https:// babel.hathitrust.org/cgi/pt?id=umn.31951d02987449r;view=1up;seq=3. 
Nutrition Labeling in the United States and the Role of Consumer Processing, Message Structure, and Moderating Conditions

Williams, P. (2005). Consumer understanding and use of health claims for foods. Nutrition Reviews, 63(7), 256-264.

\section{Additional Resources}

FDA. (2016). Guidance for industry: A food labeling guide (7. Nutrition labeling; Questions G1 through P8).

FDA. (2016). Labeling \& nutrition.

FDA. (2016). Label claims.

Food and Nutrition Information Center. (2016). Food labeling.

National Heart, Lunch, and Blood Institute. (2013). Use the nutrition facts label.

Nutrition. (2016). Food labels.

USDA. (2016). Overview of nutrition labeling in the United States and Canada.

\section{J. Craig Andrews}

Professor and Charles H. Kellstadt Chair in Marketing, Marquette University

\section{Scot Burton}

Department of Marketing Transportation, University of Arkansas

\section{Laurel Aynne Cook}

Department of Marketing, West Virginia University

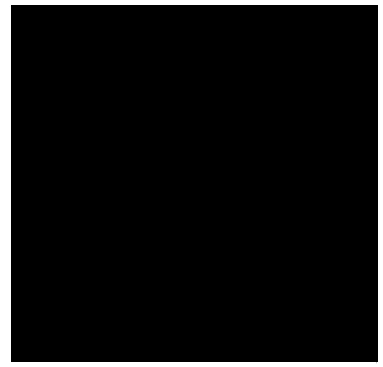

